\title{
Developments in mycotoxin analysis: an update for 2020-2021
}

\author{
S.A. Tittlemier ${ }^{1}$, B. Cramer ${ }^{2}$, C. Dall'Asta ${ }^{3}$, M.C. DeRosa ${ }^{4}$, V.M.T. Lattanzio ${ }^{5}$, R. Malone ${ }^{6}$, C. Maragos $^{7}$, M. Stranska $^{8}$ \\ and M.W. Sumarah' \\ ${ }^{1}$ Canadian Grain Commission, Grain Research Laboratory, 1404-303 Main St, Winnipeg, MB, R3C 3G8, Canada; \\ ${ }^{2}$ Westfälische Wilhelms-Universität Münster, Institute of Food Chemistry, Corrensstr. 45, 48149 Münster, Germany; ${ }^{3}$ Università \\ di Parma, Department of Food and Drug, Viale delle Scienze 27/A, 43124 Parma, Italy; ${ }^{4}$ Department of Chemistry, Carleton \\ University, Ottawa, ON, K1S 5B6, Canada; ${ }^{5}$ National Research Council of Italy, Institute of Sciences of Food Production, \\ via Amendola 122/O, 70126 Bari, Italy; ${ }^{6}$ Trilogy Analytical Laboratory, 870 Vossbrink Dr, Washington, MO 63090, USA; \\ ${ }^{7}$ United States Department of Agriculture, ARS National Center for Agricultural Utilization Research, Peoria, IL 61604, \\ USA; ${ }^{8}$ Department of Food Analysis and Nutrition, Faculty of Food and Biochemical Technology, University of Chemistry \\ and Technology, Technicka 5, Prague, 166 28, Prague, Czech Republic; ${ }^{9}$ Agriculture and Agri-Food Canada, London Research \\ and Development Centre, 1391 Sandford Street, London, ON, N5V 4T3, Canada; sheryl.tittlemier@grainscanada.gc.ca
}

Received: 8 November 2021 / Accepted: 4 February 2022

(c) 2022 Wageningen Academic Publishers

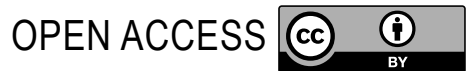

REVIEW ARTICLE

\begin{abstract}
This review summarises developments published in the period from mid-2020 to mid-2021 on the analysis of a number of diverse matrices for mycotoxins. Notable developments in all aspects of mycotoxin analysis, from sampling and quality assurance/quality control of analytical results, to the various detection and quantitation technologies ranging from single mycotoxin biosensors to comprehensive instrumental methods are presented and discussed. The summary and discussion of this past year's developments in detection and quantitation technology covers chromatography with targeted or non-targeted high resolution mass spectrometry, tandem mass spectrometry, detection other than mass spectrometry, biosensors, as well as assays using alternatives to antibodies. This critical review aims to briefly present the most important recent developments and trends in mycotoxin determination, as well as to address limitations of the presented methodologies.
\end{abstract}

Keywords: sampling, multi-mycotoxin analysis, quality control, multiplex, biosensor, chromatography, non-targeted analysis, aptamer, ELISA, molecularly imprinted polymer, high resolution mass spectrometry, tandem mass spectrometry

\section{Introduction}

This article is the latest instalment in a series of annual reviews highlighting analytical method developments for mycotoxin determination, continuing from the previous review covering the mid-2019 to mid-2020 period (Tittlemier et al., 2021). As with the previous reviews in this series, our primary purpose is to raise awareness of notable developments and advances in the analysis of mycotoxins and to highlight where improvements can help the wider 'mycotoxin community' of analysts, researchers, regulators, and those relying on analytical data. This review is not meant to be an exhaustive list of publications on mycotoxin analytical methods.
We have continued with organising the review to cover sampling (section 2), quality control of mycotoxin analyses (section 3), chromatography with tandem mass spectrometry (MS/MS; section 4), chromatography with targeted high resolution mass spectrometry (HRMS; section 5), chromatography with non-targeted HRMS (section 6), chromatography with non-mass spectrometric (MS) detection (section 7), multiplex biosensors (section 8), single mycotoxin or single mycotoxin family biosensors (section 9), and assays using antibody analogues (section 10).

While many valuable advances were published over the past year, contributing authors from most sections of the review noted the continued absence of important details in many publications. In particular, the absence 
of specific information on sampling and processing equipment and procedures used, method sensitivity reported on a sample mass basis, and the application of novel detection technologies to 'real' matrices continued in many publications. Authors, reviewers, and editors need to ensure that this information is communicated thoroughly and clearly, in addition to ensuring reported results are based on sound science. In order to promote this valuable communication approach, the World Mycotoxin Journal is planning to update its instructions to authors and reviewers to minimise the publication of articles lacking important information.

\section{Sampling}

Research on sampling and sample processing published over the past year include characterisation of mycotoxin distributions among maize kernels (Chavez et al., 2022) and within fields (Yi et al., 2021), an enhanced analysis of existing data sets on mycotoxin spatial distribution in bulk volumes (Kerry et al., 2021), and descriptions of sampling techniques for blood (Vidal et al., 2021), grain dust (Limay-Rios and Schaafsma, 2021), and for pooling samples for mycotoxin screening (Cheng et al., 2020). A very welcome retrospective analysis was also published on the disappointing decrease in researchers' consideration of proper sampling and processing of maize (Kumphanda et al., 2021).

Kumphanda et al. (2021) reviewed papers on the analysis of mycotoxins in maize published in 1991 through 2020. In particular, they focused on the laboratory sample mass (amount of maize comminuted) and test portion mass (amount of ground maize extracted). The results of their review were disheartening. Only $50 \%$ of publications explicitly stated the laboratory sample mass used, greater than $67 \%$ of publications did not specify the type of grinder and sieve size used to comminute grain, and laboratory sample and test portion masses both showed a statistically significant decline over the three decades reviewed. The authors proposed that high solvent costs, increased time needed for sample preparation and processing, and increased resources needed to dispose of excess sample, all contributed to the decrease in laboratory sample and test portion sizes at the expense of increasing total variance of test results. In addition to the journal editors and food safety bodies challenged to uphold the quality and reporting of sampling processes by the authors, funding bodies and reviewers also need to hold researchers accountable for using sound sampling and sample preparation and for clearly providing this necessary information in publications.

As part of their work on improving high throughput UVvis-near-infrared (NIR) screening of maize kernels for contamination with aflatoxin and fumonisins, Chavez et al. (2022) generated a substantial data set on concentrations in individual kernels. These data reinforce the constitution heterogeneity of aflatoxins and fumonisins in bulk maize (i.e. differences in amount of mycotoxin between kernels), as there were low frequencies of high concentrations in single kernels. Even though the selection of kernels for analysis from the bulk samples was biased towards those kernels displaying risk factors associated with the presence of mycotoxins, only 1 and $5 \%$ of kernels contained aflatoxins $\geq 20 \mu \mathrm{g} / \mathrm{kg}$ and fumonisins $\geq 2 \mathrm{mg} / \mathrm{kg}$, respectively. The aim of the biased selection was to produce a set of test materials to facilitate development of the screening prediction model. Interestingly, estimates of aflatoxin and fumonisin concentrations in bulk maize made from the targeted individual kernel results showed no bias when compared to the concentrations obtained from analysis of a $250 \mathrm{~g}$ sub-sample taken from the bulk samples. The lack of apparent bias is most likely reflecting very high kernelto-kernel differences, that overshadowed any effect from kernel selection bias.

Yi et al. (2021) sampled two fields of wheat and rice and performed a spatial analysis of deoxynivalenol (DON) and aflatoxins, respectively. The fields were segmented into three sections (middle, peripheral, and 'other' - the span between the middle and the periphery of the fields). The authors reported a 'filtration effect' by the peripheral section, with more dust, fungal spores, and mycotoxins on grain collected from this section. The reported results emphasise the need to consider spatial variation within a field when sampling plant material for mycotoxin analysis. Unfortunately, no methodological details were given on the fungal analyses, and the description of sampling was also severely lacking. There was no information provided on the mass of grain collected for samples; no information on the tools and procedures used for comminution of whole grain, nor the particle sizes obtained; and no information on how the comminuted grain was sub-sampled. The lack of methodological details prevent full consideration of the results. However, the purported conclusions could be used to develop more robust field studies on the situational factors that impact field-sampling for mycotoxin analysis.

Kerry et al. (2021) re-visited spatial analyses of DON and ochratoxin A (OTA) in a truckload of wheat and fumonisins in a maize pile. Because of the heterogeneity of OTA and fumonisins in the grain studied, the highly skewed concentrations obtained through previous testing were transformed to normal scores to properly investigate the spatial correlation of the mycotoxins within the wheat truckload and maize pile. Different from the previous analysis (Rivas Casado et al., 2009), Kerry et al. found that OTA showed clustering within the truckload. The authors also suggested that understanding spatial variability of bulk volumes could improve accuracy and reduce the cost of sampling plans, but unfortunately did not discuss how consistent (or not) the spatial distribution of DON, OTA, 
and fumonisins would be amongst different truckloads, piles, and other bulk volumes of grain.

Other research published over the last year investigated sampling techniques. Vidal et al. (2021) reported on 'volumetric absorptive microsampling' (VAMS) as an alternative to dried blood spot sampling. With VAMS a fixed volume of blood is absorbed by a polymeric absorbent tip, which is subsequently dried and extracted. This blood sampling technique avoids the challenges with spreading and associated heterogeneity issues when hole punch sampling dried blood spots. The authors provided details on the absorbing and extraction parameters of VAMS used in their method, and detected similar concentrations of a number of mycotoxins present in whole blood [OTA, ochratoxin $\alpha(\mathrm{OT} \alpha)$, zearalenone (ZEN), aflatoxin $\mathrm{B}_{1}$ $\left.\left(\mathrm{AFB}_{1}\right)\right]$ using VAMS and an existing validated liquid-liquid extraction method. Stability assessment showed absorbed whole blood samples were stable after 1 and 3 weeks of storage, suggesting VAMS is a robust sampling method that can be a useful tool when analysis of whole blood samples cannot take place immediately after sampling.

Limay-Rios and Schaafsma (2021) reported on research that measured a number of mycotoxins in paired whole grain winter wheat and grain dust samples collected during unloading of combines during harvest and unloading of grain storage bins into trucks. The grain flow was manually sampled using a cup, and dust was sampled using a modified commercially available vacuum cleaner during the entire period of grain sampling near the point the grain flow was deposited. Concentrations of 25 out of the 26 mycotoxins detected were greater in dust than in whole grain (by factors of 3 to 62), with only deoxynivalenol-3-glucoside (DON-3G) at higher concentrations in the whole grain, indicating dust may be a good matrix for screening for the presence of many mycotoxins. The authors reported strong correlations between dust and grain concentrations of OTA $\left(r^{2}=0.976\right)$ and DON $\left(r^{2}=0.949\right)$, and discussed how models developed from their data can be used to predict grain mycotoxin concentrations in some cases but that variability in the dust/grain relationship limits the application of the model to more stringent specifications, such as $1000 \mu \mathrm{g} / \mathrm{kg}$ DON in wheat. The low concentrations and detection frequencies observed precludes use of the model to predict concentrations in grain for the other mycotoxins included in the study.

Cheng et al. (2020) examined pooling strategies to reduce the cost of mycotoxin screening by 48 - and $96-$ well enzyme-linked immunosorbent assay (ELISA) tests using simulations incorporating the maize single kernel aflatoxin and fumonisin data from Chavez et al. (2022). The authors modelled outcomes of combining extracts of multiple samples and analysing the composite extracts to quickly rule out negative samples and assessed if the reduction in numbers of analyses gained by pooling was offset by increased cost in supplies due to the complexity of pooling strategies. Considering two-dimensional 48-well ( 6 rows $\times 8$ columns) and 96 -well ( 8 rows $x 12$ columns) ELISA plates, four pooling strategies were examined: (1) one-dimensional 'by rows' (pooling of 8 or 12 samples), (2) one-dimensional 'by columns' (pooling of 6 or 8 samples), (3) two-dimensional (pooling of samples by considering results from all relevant columns and rows), plus (4) Shifted Transversal Design ('layers' of sample combinations are constructed and varied to minimise the number of times any two samples appear together in a pool). Cheng et al. concluded that one-dimensional and Shifted Transversal Design pooling minimise reagent costs, taken as the relative number of tests needed to identify positive individual samples as compared to the scenario of no pooling, by 70 and $80 \%$, respectively, but only when the expected frequency of analyte prevalence in samples is below $21 \%$ (for 48 well plates) and $<13-21 \%$ (for 96 -well plates). In these two pooling strategies, pipetting costs increased up to a factor of 4.3-fold. The authors did not assign monetary value to costs of reagents, other consumables, and personnel time in order to estimate total savings as laboratories may value these expenditures differently.

\section{Quality control of mycotoxin analyses}

To guarantee a uniform application of regulations for mycotoxins, harmonisation and verification of standardised methods for analysis of mycotoxins is of the uppermost importance. From mid-2020 to mid-2021, results of two collaborative studies focused on validation of methods in support of EU regulations as requested by the M/520 standardisation mandate of the European Commission, were published. The first study of De Girolamo et al. (2020) addressed the fourth item of the standardisation mandate (EC, 2013), aiming at optimising and validating an analytical method for the simultaneous determination of nivalenol (NIV), DON, 3-acetyl-deoxynivalenol (3-ADON), 15-acetyl-deoxynivalenol (15-ADON), T-2 toxin (T-2), HT-2 toxin (HT-2), and ZEN in cereals and cereal products by liquid chromatography with tandem mass spectrometry (LC-MS/MS). Sample was extracted using acetonitrile/ water 84/16 (v/v), and the extract was analysed after solid phase extraction (SPE; using Oasis ${ }^{\circ}$ HLB; Waters, Milford, MA, USA) clean-up. Data from the collaborative study, as well as feedback from participants, confirmed that the chromatographic separation of 3-ADON and 15-ADON remains one of the most challenging steps of the method. At the same time, lower (71-78\%) recoveries were observed for NIV. These were still deemed 'acceptable' by the authors; unfortunately, the benchmark for 'acceptable' was not discussed. Despite this, the results of the collaborative study met the required method performance criteria with HorRat values $<2.0$ and have been recently adopted as a CEN standard (EN17280:2019). 
The second study of Tangni et al. (2021) was focused on determination of CIT in food by LC-MS/MS, as a response to the eleventh priority of the M/520 standardisation mandate. Within this validation study, in-house prepared matrix reference materials of red yeast rice, wheat flour, and Ginkgo biloba leaves were used, which is in line with current trends preferring the matrix reference materials with incurred mycotoxins, over the reference materials prepared using fortification (Tittlemier et al., 2021). From all of the tested matrices, Ginkgo biloba was reported to be problematic by some participants (in particular, incorrect CIT ion rations, as well as influencing of the signal of the ${ }^{13} \mathrm{C}$-labelled internal standard and native CIT were observed in this matrix), indicating that increased attention should be paid when analysing Ginkgo biloba-based products. Nevertheless, evidenced by HorRat values $<2.0$, the results of the collaborative trial demonstrated that the applied analytical method could be standardised for all of the matrices, at levels considered by the European Commission for setting regulatory limits (Tangni et al. 2021).

Çetinkaya and Çetinkaya (2021) published a paper introducing a new proficiency testing (PT) scheme suggested by the experienced PT organiser, Turkish National Food Reference Laboratory. This institution has eight years of experience with organising PTs focused on determination of AFs and OTA, as the most frequent mycotoxins in Turkish export products (dried fruits, pistachio, hazelnuts, and others). Instead of the generally used PT scheme utilising the Horwitz-Thompson model to obtain the standard deviation for z-score calculation (Thompson 2000), the newly suggested scheme introduced the possibility of calculation a 'robust standard deviation' by the Q/Hampel method (ISO, 2015). The results from previous rounds of PTs obtained between the years 2013 and 2020 were utilised to recalculate and compare results from both evaluation principles. Contrary to the Horwitz-Thompson model, where the relative standard deviation (RSD) is set to $22 \%$ for concentrations below $120 \mu \mathrm{g} / \mathrm{kg}$ (this value has been set empirically based on observations from many collaborative trials of many parameters over an extended time period), the new model calculates the standard deviation from a more homogenous data set (results of specific mycotoxins from specific matrices). The authors claimed use of the Q/ Hampel model would provide more realistic results, but did not elaborate on this claim. The recalculated 'robust standard deviation' was $17 \%$. The results of the study showed that reducing RSD from 22 to $17 \%$ narrowed the limit values and satisfactory range of $\mathrm{z}$-score by about $30 \%$. Despite the fact that a more strict RSD limits the success rate of the participants and is more challenging for the laboratories, it is beneficial in terms of improved quality assurance of the mycotoxins analysis (Çetinkaya and Çetinkaya 2021).

The authors of the study also mention that nowadays, many PT organisers frequently use the low-cost approach of calculating a mean value from participants' results for generating the assigned value, without any additional measurements (i.e. of a certified reference material). They point to the fact that this approach suffers from the rather high risk of biased results when the consensus of participants' results is low (Çetinkaya and Çetinkaya 2021). The topic of non-conformity of PT results and influence on the assigned value was previously discussed in study of Zachariasova et al. (2014), where bimodal distribution of results for DON and ZEN in cereals was observed. In this particular case it was caused by the detection technique used by the laboratories, and overestimation of results obtained by immunoassays cross-reacting with DON and ZEN 'masked' conjugates and matrix interferences, when compared to results of liquid chromatography mass spectrometry methods (Zachariasova et al., 2014). This demonstrates that the design of the PT is essential, and new contributions to this topic may be inspirational, and lead to improvements in quality control of generated results and improved performance standards in analytical laboratories. This study also emphasises, even though there is an increased cost, one of the best ways of assigned value generation is by utilising isotopically labelled mycotoxins as internal standards, which are able to effectively manage the matrix effects and achieve a satisfactory precision (Tittlemier et al., 2021).

The last paragraph of this sub-chapter is devoted to quality assurance in conjugated mycotoxins analysis, as the gradually increasing area of interest of the mycotoxin analysts' community. The biggest obstacle in accurate analysis and quantification of mycotoxin conjugates is the limited commercial availability of analytical standards. To date, only some mycotoxin conjugates can be purchased in sufficient purity. In addition to well-known DON-3G from Sigma Aldrich (St. Louis, MO, USA), conjugates of ZEN and its metabolites as e.g. zearalenone-14-glucoside (ZEN-14G), zearalenone-14-sulfate (ZEN-14S), zearalenone-14,16disulfate (ZENdiS), $\alpha$-zearalenol-14-glucoside $(\alpha-Z E L$ $14 \mathrm{G}), \alpha$-zearalenol-14-sulfate $(\alpha$-ZEL-14S), $\beta$-zearalenol14 -glucoside ( $\beta$-ZEL-14G), $\beta$-zearalenol-14-sulfate $(\beta$-ZEL-14S), and zearalanone-14-glucoside (ZAN-14G) can be purchased from ASCA GmbH (Berlin, Germany). These eight conjugates were incorporated into the multianalyte method for determination of mycotoxins and their metabolites in beer (Rausch et al., 2021).

It is also worth mentioning biotransformation by plants or microorganisms as another option of gaining these rare and rather expensive mycotoxin conjugates. Recently, Peters et al. (2021) introduced an easily implementable microbiological method for production of ZEN glycosides. This was achieved by Cunninghamella fungal strains in sulphate-depleted media, where the lack of sulphur steered the reaction towards the preferred abundant production of ZEN-14G and zearalenone-16-glucoside (ZEN-16G). 
Interestingly, when the developed approach was applied for other mycotoxins (namely DON, $\mathrm{AFB}_{1}, \mathrm{FB}_{1}, \mathrm{~T}-2$, and OTA, no obvious biotransformation was observed at the conditions previously used for ZEN (Peters et al., 2021) indicating that different microorganisms have specific metabolic pathways for detoxification of different mycotoxins. For checking the concentrations of standards of conjugated mycotoxins, measurement of absorbance and calculation of concentrations via the molar absorption coefficients, as described by Scheibenzuber et al. (2021) is highly recommended. Scheibenzuber et al. (2021) also describe a method for determining molar absorption coefficients of previously uncharacterised conjugated mycotoxins.

\section{Chromatography with MS/MS}

As already described in the last updates (Tittlemier et al., 2020, 2021), the trend towards the development of multitoxin methods is still rising, as attested by the number of reports published over the past year. The growing interest of policy-makers around multiple chemicals in food, has supported the expansion of multi-analyte protocols, able to detect different groups of contaminants in the same food matrix (i.e. mycotoxins and pesticides in vegetables, or mycotoxins and veterinary drugs in food from animal origin).

While the application of LC-MS/MS methods to mycotoxins in regulated matrices is almost routinely performed, and therefore less novelty can be expected in that field, several protocols have been developed for unusual matrices, thus offering a valuable contribution in the assessment of emerging risks. Two papers published over this year can be regarded as the real breakthrough in the use of LC-MS/ MS for mycotoxin analysis.

Steiner et al. (2020) presented the first multiclass quantitative method for the determination of more than 1,200 biotoxins and other contaminants in complex feed. The efforts in the optimisation of the chromatographic separation together with the appropriate MS/MS conditions, have allowed the accurate quantification of compounds with a wide range of physico-chemical properties. All the quality parameters fall within the EU validation guidelines (EC, 2006). Validation data provided have confirmed matrix effects as the more critical control point of the entire procedure. Although the inter-laboratory transferability of such protocol could be cumbersome due to the number of parameters to keep under control, it provides a strong proof of concept about the potential of the most advanced LC-MS/MS instrumentation for multiclass analysis. In consideration of increasing interest in combined effects of multiple toxicants (EC, 2020), this study provides a first reliable tool for the assessment of co-exposure from multiple contaminants in animal feed.
The same group was also involved in a validation study offering relevant insight into the parameters to control for accurate quantification of multiple contaminants (Sulyok et al., 2020). In addition to the value of the data provided, the paper is a blueprint for further validation studies of multiclass protocols. In particular, the authors underlined the importance of a thorough evaluation of matrix effects, which can vary a lot among chemical classes, and may affect final results.

As already said, several papers have addressed the occurrence of mycotoxins in unusual food matrices, or have enlarged the number of mycotoxins potentially detected in usual food matrices. In this context, an interesting example is the multi-toxin determination of regulated, emerging and modified mycotoxins in milk proposed by González-Jartín et al. (2021). The authors validated a method for the accurate quantification of 40 analytes in milk, using a QuEChERSbased extraction. Although aflatoxin $M_{1}\left(A F M_{1}\right)$ is the only mycotoxin regulated in milk so far, protocols able to provide accurate data about the potential occurrence of other mycotoxins are needed for a more informed risk assessment. In particular, the authors reported on the frequent occurrence of enniatins and beauvericin (BEA) in milk, although at low concentrations. It should be noted that, in spite of the large range of chemically diverse analytes and the complex matrix, no internal standard was used. This might affect the overall analytical performance of the method, especially when a large number of samples is screened over time.

As for infant food monitoring, Braun et al. (2021) developed a multi-toxin strategy based on two complementary methods, covering 46 mycotoxins overall in infant formulae and meals. In particular, the authors adopted two LC-MS/ MS methods targeting two sets of mycotoxins, the former covering the most common Aspergillus, Penicillium, and Fusarium mycotoxins, and the latter considering Alternaria mycotoxins. Since the study focused on infant food, the need to achieve a very high sensitivity did not allow for any compromise in terms of chromatographic separation and matrix effect, thus requiring the use of two separate protocols of analysis. Due to the lack of certified reference materials and the large variety of food components used for infant food formulation, no matrix-matched calibration was used. Satisfactory validation parameters were obtained using a thorough standard calibration followed by spiking experiments, although 12 analytes were beyond the satisfactory recovery range 50-150\%; likely due to strong matrix effects. The sensitivity range was good, with limits of detection (LODs) ranging between $0.1 \mu \mathrm{g} / \mathrm{kg}$ for aflatoxicol, a metabolite of $\mathrm{AFB}_{1}$, and $12 \mu \mathrm{g} / \mathrm{kg}$ for tenuazonic acid (TeA). According to the data collected, trace levels of 17 mycotoxins were found in samples from the market $(n=59)$, among them the previously unreported sterigmatocystin and aflatoxicol. 
Although beer has been often targeted for mycotoxin analysis, the method proposed by Rausch et al. (2021) provided a very comprehensive overview of potentially occurring parent and modified forms. Beer being a complex matrix, the extraction and clean up procedures reported in the literature are commonly SPE, immunoaffinity, or QuEChERS based. On the contrary, the authors proposed a solvent-precipitation strategy, using acetonitrile as precipitation agent. Such simple clean up, although timeand cost-convenient, led to a strong matrix effect for many analytes ( $-67 \pm 319 \%)$, compensated for by using zearalenonedimethylether- $\mathrm{D}_{6}$ as an isotopic-labelled internal standard. The protocol returned validation parameters compliant with the EU guidelines (EC, 2006). In particular, sensitivity [limits of quantitation (LOQs) $0.04-75 \mu \mathrm{g} / \mathrm{l}$ ] and recovery $(79-100 \%)$ were highly satisfactory for all the analytes.

Rämö et al. (2021) applied LC-MS/MS for the accurate quantification of Fusarium toxins in onions. It is actually known that Fusarium species may cause a disease called Fusarium basal rot in onion, but the possible occurrence of mycotoxins in the edible tissues is still understudied. While a dilute-and-shoot approach was applied for fumonisins and BEA with a minor matrix effect, a concentration step was required for the quantification of moniliformin (MON). ${ }^{13} \mathrm{C}-\mathrm{FB}_{1}$ was used as an isotope-labelled internal standard. The method was able to detect the main fumonisins $\left(\mathrm{FB}_{1}\right.$, $\mathrm{FB}_{2}$, and $\mathrm{FB}_{3}$ ) together with $\mathrm{MON}$ and BEA with good recoveries (67-122\%) and sensitivity (LOQs: $2.5-10 \mu \mathrm{g} / \mathrm{kg}$ ).

Fruit juice and purées are a food category of interest for the development of multi-analyte methods, in consideration of the reported increasing fungal infections due to climate change. Guo et al. (2021) presented a LC-MS/MS protocol for the quantification of 15 major mycotoxins produced by Aspergillus, Penicillium and Alternaria species in orange, grape, and apple juices. The extraction procedure was based on the use of QuEChERS, and allowed for satisfactory recoveries (74-110\%) and sensitivity (LODs: 0.05-0.1 $\mu \mathrm{g} / \mathrm{l}$ ). Matrix-matched calibration was used to compensate for matrix effects. The study demonstrated frequent occurrence of mycotoxins in juices from the market, thus confirming the need for further studies and proper monitoring.

A very innovative application of the multi-toxin approach was reported by Goessens et al. (2021), who developed a method for the determination of 20 mycotoxin in freshwater. The authors started from the observation that mycotoxins can leach out of plant tissues and be transported through runoff water into ponds and water basins. In view of the increasing number of extreme climate events, the potential role of mycotoxins in ecotoxicology has to be considered. Due to the very low expected amounts, the authors applied an SPE clean-up and concentration step, coupled with the use of isotope-labelled internal standards. Satisfactory data were collected for matrix effects, extraction recoveries
(76-110\%), RSD (18\%), and LOQs (1-40 ng/l) with the only exception of alternariol monomethyl ether (AME), which required a matrix-matched calibration curve. In terms of sample storage and preparation, the authors reported on the possible microbial degradation of analytes over time, and therefore the need to perform the analysis no longer than 96 hours after sample collection. The method was applied for the analysis of freshwaters from ponds across Flanders, Belgium. Enniatins and BEA, together with AME, ZEN and the more potent oestrogenic compound $\alpha$-ZEL were frequently found in water.

A novel approach in terms of extraction has been reported by Gbashi et al. (2020). The authors proposed the use of pressurised hot water for the extraction of 15 different mycotoxins from cereals. By means of advanced chemometrics, results obtained by conventional extraction and pressurised hot water extraction were compared, finding a good consistency. The application of green extraction strategies for multiclass analysis is relevant, as it may improve the environmental sustainability of the protocols and may offer an alternative for those areas where solvent storage may be an issue.

Taken altogether, these examples reflected the large variety of food matrices analysed so far by LC-MS/MS for multiple mycotoxins. It is clear that the scientific community is more and more interested in capturing mycotoxins co-occurrence in major crops, as well as in understanding the potential accumulation of unregulated mycotoxins in unregulated commodities. A few papers also addressed environmental matrices, such as soil and water. For accurate quantification, targeted LC-MS/MS is still the benchmark thanks to the large affordability and the robustness of the technique. However, a thorough validation workflow, and in particular a careful assessment of the matrix effect, is still somehow missing due to the lack of certified reference materials and calibrants. In terms of sample preparation, many reports are based on QuEChERS approach, on account of their ease in storage and handling. A growing number of papers are focused on the application of green strategies, with a general trend in decreasing the use of solvents. The use of isotopelabelled standards is growing, although still not routine.

Besides the breakthrough protocols proposed by Steiner et al. (2020) and Sulyok et al. (2020), a vast majority of less sophisticated, but still efficient, methods are still in use, offering a good compromise between the affordability of the analytical system and the quality of the analytical performance.

\section{Chromatography with targeted HRMS}

High resolution mass spectrometry (HRMS) has found its place in many different research areas, being a core analytical technique for many omics applications. For 
targeted analysis, HRMS is becoming more and more popular, as well. The improved selectivity due to high resolution in combination with the fast generation of product ion spectra can reduce the number of ambiguous results and streamline peak detection. However, regarding the limits of detection and quantitation (LOD, LOQ), a gap between HRMS and MS/MS remains for most analytes. To overcome this gap and broaden applicability for mycotoxin trace analysis, different analyte enrichment strategies such as solid phase extraction have been implemented in workflows. Unfortunately, these additional steps always cause a strong increase of manual laboratory work and costs, thus reducing possible benefits of HRMS systems.

Online SPE as an alternative to classical SPE allows users to omit additional lab work by installing a re-usable SPE column into a valve unit placed into the ultra-high pressure liquid chromatograph (UHPLC) flow path ahead of the chromatographic column. When in load position, the SPE is not connected with the chromatographic column, but sample extracts are loaded onto the column by means of a standard UHPLC autosampler. Additionally, washing of the SPE column with appropriate solvents or solvent combinations is possible. Switching the valve unit to elute mode places the online SPE column into the main flow path and the concentrated, purified extract is reversely eluted from the SPE onto the chromatographic column for separation. Materials of different selectivities are available for online SPE, including most standard SPE phases but specific turbulent flow SPE columns can also be used. Turboflow means that columns with narrow diameter and large particle sizes are applied, with the idea, that large (macro) molecules, due to their slow diffusion, are less adsorbed to the particles compared to small analyte molecules such as mycotoxins. As a consequence, additional purification and removal of macromolecules is achieved. Ndaw et al. (2021) investigated the applicability of the cyclone P (ThermoFisher Scientific, Waltham, MA, USA) turboflow online SPE cartridge, for the detection of mycotoxins and mycotoxin biomarkers in urine. Subsequently, they compared offline SPE with two different cartridges as an alternative manual enrichment and purification technique with the system. The authors observed that the highest sensitivity could generally be achieved with online SPE, but retention of DON was not possible with the applied material. Thus, for optimised sensitivity, the authors had to split the method into an online-SPE method for $\mathrm{AFM}_{1}, \mathrm{FB}_{1}$, ochratoxins, HT-2 and $\mathrm{ZEN}$ and second single analyte method using offline SPE on an Oasis HLB column for DON detection. Phase 2 metabolites of mycotoxins were only indirectly detected after enzymatic cleavage. Using this sample enrichment, LOD and LOQ values comparable to current MS/MS dilute and shoot approaches for most analytes could be obtained, demonstrating that the application of HRMS instruments for human biomonitoring of mycotoxins is possible. However, even modern MS/MS systems are still not sensitive enough to measure the baseline exposure to many mycotoxins. Thus, a combination of MS/MS with online SPE can also be a valuable tool, as described by Schmidt et al. (2021).

Another HRMS-based biomonitoring method for the detection of citrinin (CIT) and dihydrocitrinone has been published by Narváez et al. (2021). In this paper, an interesting chromatography is applied which seems to elute analytes in a 'wave' of methanol from a reversed phase Luna Omega series column (Phenomenex, Torrance, CA, USA). Water and methanol containing $5 \mathrm{mM}$ ammonium formate and $0.1 \%$ formic acid were applied as solvents and the analytes were eluted between 4.75 and $5.00 \mathrm{~min}$. The gradient started with $0 \%$ methanol and increased to $95 \%$ methanol within $1 \mathrm{~min}$ which was held for 0.5 min before the methanol content dropped to $75 \%$ within $2.5 \mathrm{~min}$ and $60 \%$ in the subsequent minute. At the end, the column was equilibrated with $0 \%$ methanol for $1.5 \mathrm{~min}$ prior to the next injection. The effect of this fast increase high elution power that afterwards dropped during the whole chromatographic run is not further discussed by the authors. Whether some HILIC-type interactions are taking place during the run is not clear, but the gradient itself surely requires special emphasis to the performance of the UHPLC system and transferability to other UHPLCsystems will probably be difficult.

In the last decade, ion mobility spectrometry devices based on travelling wave (Waters), drift tube ion mobility (Agilent, Santa Clara, CA, USA) and trapped ion mobility (Bruker, Billerica, MA, USA) became available as parts of enhanced ToF-HRMS Systems, providing an extra dimension of separation and selectivity. The core idea of all of these technologies is that compounds can be separated based on their collisional cross section (CCS). While the idea of CCS values is rather old and, for instance, has been implemented in screening devices for chemical warfare agents, its application and meaningfulness for compound identification in combination with mass spectrometry is currently under discussion. Especially when coupled with HRMS instruments, CCS data can improve selectivity and, depending on the system and matrix, lower the LOQ and LOD for mycotoxin detection. Following their 2018 publication on the determination of CCS values of mycotoxins for travelling wave systems, Righetti et al. (2020) now provided data on the reproducibility of CCS values among different travelling wave instruments from the same vendor. In the study three instruments from two different platforms were compared, resulting in $96.4 \%$ of the CCS values differing only by up to $2 \%$ between laboratories while for single labs, an average RSD as low as $0.15 \%$ was reached. Thus, CCS seems to be a very reproducibly measurable molecular property. Additionally, characteristic differences between CCS of isomers can be used for identification and 
probably reduce the requirements for UHPLC separation. Currently a bottleneck of the applied instruments in some circumstances seems to be the low overall sensitivity for a large number of analytes, the lowest concentration where a CCS value could be assigned was around $10 \mathrm{ng} / \mathrm{ml}$ in solvent. For most instruments, when the ion mobility separation mechanism is activated, there is a reduction in signal intensity that can affect CCS value characterisation. In the analysis of samples, this reduction in signal intensity can be more than offset by the alleviation of the negative impact from interfering compounds. In the future it will be interesting to see the sensitivity of other ion mobilityquadrupole-time of flight (Q-ToF) instruments and to what extend the additional selectivity can help to identify and quantify mycotoxins in complex matrices.

\section{Chromatography with non-targeted HRMS}

LC-HRMS for targeted analysis has become more mainstream as reviewed above with the increased availability of Q-ToF and Orbitrap instruments and the added benefits that they provide. Complementary nontarget methods such as data-independent acquisition (DIA), data-dependent acquisition (DDA) and all ion fragmentation (AIF) have also greatly increased in many fields of science including metabolomics and the study of environmental contaminants. However, a similar increase in non-targeted analysis (NTA) has not been observed for the investigation of mycotoxins. Steiner et al. (2021) provided a useful commentary on this subject in their 'trend' article on the 'Challenges and future directions in LC-MS-based multiclass method development for the quantification of food contaminants.' They identified a number of strengths of non-targeted HRMS analysis, including the ease of adopting existing targeted HRMS methods for NTA and the enhanced sensitivity for ultratrace analysis. Additionally, retrospective analysis of non-targeted data enables the possibility of identifying additional metabolites and transformation products of important mycotoxins that would otherwise be missed. They also identified two important impediments to wider uptake of this technology by the mycotoxin community. The first is that the linear working range of these instruments needs to be expanded to meet the needs of routine analysis. The second is the cost of the instruments makes them prohibitive for many labs. The key take away message is that in order for multiclass analyte screening methods to increase beyond 1000 target compounds, HRMS and NTA will be necessary. Since the previous review in mid-2020, there have been a few significant papers describing advances in NTA with HRMS for mycotoxin determination. These papers describe analysis of ergot alkaloids from grain, Alternaria metabolites in tomatoes, and mycotoxins in milk.
The European Commission (EC) recently introduced new rules limiting 12 'major' ergot alkaloids in certain food products for infants and young children (EC, 2021). A timely study by Uhlig et al. (2021) applied non-targeted HRMS analysis with post-acquisition diagnostic fragment filtering (DFF) to unravel the complexity of ergot alkaloids and indole diterpenoids in sclerotia (Uhlig et al., 2021). Analysis was performed on samples obtained from wild grasses, rye and oats from Norway or Canada. MS/MS datasets were screened for the ergot alkaloid specific product ions of $\mathrm{m} / \mathrm{z} 208.0748$ and 223.1230 and 130.0651 and 146.0600 for indole diterpenoids. Using this technique they were able to identify 67 ergot alkaloids and 5 indole diterpenoids from the grain samples. Interestingly, the 6 major ergot alkaloids and their epimers made up more than $50 \%$ of the total ergot alkaloids detected in samples from Canada and Norway. These data support monitoring for the $12 \mathrm{EC}$ ergot alkaloids in food and feed as being sufficient to manage risk from a food safety perspective. One additional benefit that NTA provided was insight into chemotaxonomic markers that were specific to certain species of Claviceps.

HRMS with DDA was used for the analysis of mycotoxins from Alternaria-inoculated tomatoes to assess potential risks associated with these emerging mycotoxins (Zhang et al., 2021a). The authors noted that conjugation with sulphate is a common mechanism for Alternaria compounds. In order to screen for these sulphated metabolites, data were processed using DFF set for identification of the neutral loss of 79.9568 corresponding to the loss of the sulphate. Twenty-four sulphated Alternaria metabolites were identified from fungal cultures, these data were then used to build a custom database of Alternaria mycotoxins and sulphated metabolites. Screening of Alternaria inoculated tomatoes revealed that two of these sulphated compounds [alternariol-sulphate (AOH-sulphate) and AME-sulphated] were present, indicating the potential risk of these Alternaria metabolites in food. The authors were also able to retrospectively screen for and confirm that there were no glucose conjugated metabolites from Alternaria in the tomatoes. This work demonstrated the power of combining HRMS for target analysis and NTA but could have been improved by expanding the analysis to more food products including samples that were not inoculated.

A metabolomics based approach was used to analyse for regulated and emerging mycotoxins in bulk milk samples from cows fed maize silage (Rocchetti et al., 2021). Retrospective screening identified 46 mycotoxins within milk samples that were previously placed into one of five groups based on the level of fungal contamination in the silage the cows were eating. Multivariate statistical analysis of the data resulted in clustering of the milk samples from cows fed the higher mycotoxin contaminated silage. Additionally, orthogonal projections to latent structures 
discriminant analysis and $\mathrm{k}$-means clustering revealed four discriminant marker compounds that were driving the separation of the milk samples. These four compounds could potentially be linked back to specific fungal contaminants within the feed. This work demonstrates the value of using non-targeted HRMS analysis combined with retrospective screening for assessing mycotoxin contamination.

Although NTA with HRMS for mycotoxin determination has not experienced the uptake by the community as it has in other fields, these papers clearly demonstrate its value. NTA for screening will likely continue to gain traction as databases improve and software packages for data process are better equipped to handle these large datasets. The ability to retrospectively screen for $>1000$ compounds of interest is not possible for most lab doing targeted analysis due to the cost of standards and their limited commercial availability. Alternatively, as noted by Steiner et al. (2021) the cost of these instruments will be a major factor for labs, as well as the additional knowledge required by the analyst due to the complexity of the analysis. There is a lot of discussion in other fields about how NTA will support regulation, it is expected that this will be important for mycotoxin determination, as is evident from the papers discussed here.

\section{Chromatography with non-MS detection}

Various analytical methods for mycotoxin quantification with non-MS detection were developed from the middle of 2020 to the middle of 2021 . While a vast number of analytical investigations have been undertaken, further research on non-MS mycotoxin detection is important to support laboratories that do not have the financial resources or expertise to utilise MS detection methodologies.

One of the recent advances in mycotoxin analysis was the development of an advanced pre-treatment technique for the analysis of aflatoxins in spices (Saito et al., 2020). This FLD method uses dispersive solid phase extraction (dSPE) together with solid-phase fluorescence derivatization and immunoaffinity gel to provide a simple, quick, and yet sensitive liquid chromatography technique for detecting aflatoxins. In developing the method, pepper samples (black and white) were extracted using a 4:1 v/v methanol-water mixture and then diluted using a $7 \%$ Triton $\mathrm{X}$ solution. The resulting solution was then treated with dSPE, immunoaffinity gel, and trifluoroacetic acid to yield a 0.15 $0.29 \mu \mathrm{g} / \mathrm{kg}$ solid phase fluorescence derivatization detection limit in white and black pepper with 61.7-87.8\% recovery and intermediate precision $<15 \%$ (Saito et al., 2020). The method demonstrated good performance, however the trifluoroacetic acid derivatization is not stable and this can cause complications while analysing multiple samples on one analytical sequence.
Kumar et al. (2020) undertook a similar study and developed an FLD method for analysing and validating aflatoxins in animal feeds using UHPLC without derivatisation. This technique used $80 \%$ methanol extraction with a water-based immunoaffinity column clean-up. The method was validated using a variety of feed matrices. The technique is useful in aflatoxin detection as it minimises co-extractives and matrix meddling. This UHPLC-FLD method proved a simple process for aflatoxin analysis in an extensive range of animal feed, with high sensitivity and cost-effectiveness. For instance, in pigeon pea husk feed, an LOQ of $0.5 \mu \mathrm{g} / \mathrm{kg}$ for each aflatoxin, with recoveries of $\mathrm{AFB}_{1}, \mathrm{AFB}_{2}, \mathrm{AFG}_{1}$, and $\mathrm{AFG}_{2}$ of 71.5, $75.6,82.4$, and $78.2 \%$ respectively, were obtained (Kumar et al., 2020). The development of methods for complex feed matrices with FLD continues to be important as MS-based methods with complex matrices may experience matrix specific signal suppression or enhancement.

A separate multi-mode mechanism for detecting ZEN, a highly potent oestrogenic mycotoxin in rice, was recently published (Xu et al., 2020). A hydrophilic polyhedral oligomeric silsesquioxane-containing monolithic column was prepared and coupled to high-performance liquid chromatography (HPLC) in order to detect ZEN using FLD (Xu et al., 2020). The functional and structural evaluation of the column revealed that this technique provided good selective recognition and detection of ZEN; cross-reactivity assessment showed no recognition of $A_{F} B_{1}$ and minimal recognition of $\alpha-Z E L$ (3.3\% of ZEN). Recoveries of ZEN from rice fortified at $0.2-10 \mu \mathrm{g} / \mathrm{kg}$ ranged from $99-101 \%$ with RSDs of $2-3 \%$. The authors reported an LOD of 0.02 $\mathrm{ng} / \mathrm{ml}$; unfortunately only on the basis of sample extract and not sample mass basis. Based on the sample preparation information provided, the reported LOD was calculated as $0.15 \mu \mathrm{g} / \mathrm{kg}$ on a sample mass basis.

Another method of determining mycotoxins T-2 and HT-2 in wheat grain using HPLC-FLD with 1-anthroylnitrile derivatization was recently developed and validated (Laposha et al., 2020). The method met EU method performance criteria (EC, 2006) with regards to accuracy, precision, and sensitivity. Mean recoveries for the toxins at $50-150 \mu \mathrm{g} / \mathrm{kg}$ were $91 \%$ for T-2 and $87 \%$ for HT-2 with an RSD of $0.2-4 \%$ for repeatability and intra-laboratory precision; LOQs were 2.2 and $1.2 \mu \mathrm{g} / \mathrm{kg}$ for T-2 and HT-2, respectively. The authors also reported the need to store solutions of the two mycotoxins away from light in order to avoid photodegradation.

Meanwhile, Wood et al. (2020) developed a sophisticated method for determining AFM 1 in liquid milk, cheese, and other selected milk-based proteins. The technique used automated online immunoaffinity clean-up with HPLCFLD. In developing this high-throughput approach for the quantitative analysis of $\mathrm{AFM}_{1}$, Wood and colleagues extracted $\mathrm{AFM}_{1}$ in protein products using $1 \%$ ethanoic 
acid diluted in acetonitrile with citrate salts. The AFM in the resulting extract was subsequently concentrated and purified by utilising RIDA $^{\oplus}$ CREST/IMMUNOPREP ${ }^{\circ}$ cartridges (R-Biopharm, Pfungstadt, Germany) (Wood et al., 2020). This method was found accurate and precise, with $81-112 \%$ recoveries and $4-12 \%$ repeatability. The application of automated online cartridge immunoaffinity provided added value of decreased human error during complicated extraction and purification protocols.

Belajová (2020) optimised various aspects of existing HPLC methods for the detection and quantification of the polyketide PAT and OTA in fruit purées. The study focused on infant fruit-based and fruit-cereal purées and optimised two independent detection methods: (1) diode-array and (2) fluorescence detection (FLD). The detection limits were 5.8 and $0.06 \mu \mathrm{g} / \mathrm{kg}$, for PAT and OTA, respectively. The recoveries varied between 79 and $92 \%$ for PAT and 78 and $105 \%$ for OTA.

Luci (2020) developed a method utilising enzymatic digestion with molecularly imprinted SPE extraction for quantification of OTA in pig muscle, kidney, and liver with HPLC-FLD detection. The mean OTA recovery exceeded $89 \%$ with repeatability $<5 \%$ RSD for all the matrices analysed under optimised conditions. The authors claimed an LOQ of $0.003 \mu \mathrm{g} / \mathrm{kg}$, but the lowest fortification concentration evaluated with full \% recovery and repeatability data provided was $0.1 \mu \mathrm{g} / \mathrm{kg}$. The authors reported it was possible to reuse the molecularly imprinted SPE column up to seven times without any significant peak area reduction. The study thus concluded that molecularly imprinted SPE could be used as an alternative sample preparation method for detecting OTA in pig products.

Pernica et al. (2021) assessed the effectiveness of using UHPLC-photodiode array detection (PDA) in determining the presence of PAT and potentially hazardous products formed during processing (5-hydroxymethylfurfural) in beverages. The methodology utilised MIP extraction, with separation of PAT from 5-hydroxymethylfurfural within two minutes using a Luna Omega $\mathrm{C}_{18}$ column while the wavelength of the PDA detector was set at $276 \mathrm{~nm}$. Validation parameters, including accuracy, precision, and quantitation and detection limits, were also evaluated. PAT produced a linear calibration curve from 50 to $1000 \mathrm{ng} / \mathrm{ml}$, with good linearity. LOD and LOQ for PAT, determined as the concentration of standard solution at which the signal to noise ratio equalled 3 and 10 were 4.9 and $6.6 \mu \mathrm{g} / \mathrm{l}$, respectively. The recovery rate for PAT ranged between 62 and $109 \%$, with a precision of less than $8 \%$. These findings confirmed the efficacy of UPLC-PDA in analysing PAT in the beverages sold in retail shops. Patulin method development is of continued interest due to stability issues of the toxin during sample preparation and the lack of patulin immunoaffinity columns.
Lastly, Gonçalves et al. (2020) examined the use of immunoaffinity chromatography (IAC) clean-up coupled with HPLC-post-column derivatisation-FLD in determining DON and its key conjugates in cereals. The study used IAC that interacted with DON-3G, 3-ADON, and 15-ADON for extraction, and the Hantzch reaction to form fluorescent dihydropyridine derivatives for FLD analysis. Validation variables such as capacity, cross-reactivity, and analyte competition were evaluated. The study then measured DON and its conjugates in wheat, barley, and maize fortified at $10-1000,10-300$, and $10-100 \mu \mathrm{g} / \mathrm{kg}$ of DON, DON-3G, and the two ADONs, respectively. Fifteen samples of the cereals were analysed. From the findings, the recovery rates ranged from 87 to $110 \%$. The intermediate precision was less than $14 \%$ RSD, except for DON-3G in wheat (21\%). In addition, the analysis revealed DON conjugate levels that represented up to $9-60 \%$ of the overall DON content. Since the method did not use any organic solvent when preparing the sample, it does not require additional safety precautions during sample preparation.

\section{Multiplex biosensors}

Research in the area of multiplexed immunosensors continues to grow. In recent years the greatest number of reports in this area have focused on multiplexed lateral flow immunoassays (LFIA). Increasingly, as is discussed elsewhere in this review, aptamers are also used in such devices. In addition to LFIA, a wide variety of microarrays of various formats have also been reported, with no particular format establishing dominance.

\section{Multiplexed LFIA}

Multiplexing based upon immunochromatographic devices has reached a level of maturity. Multiplexed LFIA reported in the past year included assays for detecting between two and five analytes. Most such multiplexed assays were duplex assays. In multiplex LFIA the analytes are typically distinguished from one another based upon spatial location, or with multiple labels having different colours or fluorescent properties. Improvements to LFIA generally fall into five categories: improvements to the antibodies, improvements to the competitor molecules (i.e. toxin-protein conjugates, mimotopes, etc.), improvements to the labels (brighter detection lines, etc.), improvements to detection hardware, and improvements to data analysis/ reporting. With the widespread availability of antibodies for the commercially important mycotoxins, most efforts have focused on the remaining areas.

Colloidal gold nanoparticles (AuNP) are the most common label used in LFIA. A 5-plex LFIA using AuNP was reported for application to wheat (Xing et al., 2020). An interesting feature was the use of $\mathrm{QR}$ codes to upload calibration curves into a portable reader that was used for quantification. 
Antibody-AuNP conjugates were dried onto microwells. Adding sample extract to the wells dissolved the conjugates for the competition reaction. The antibody-AuNP were captured by corresponding toxin-protein conjugates on the membrane. With the reader the limits of detection for $\mathrm{AFB}_{1}, \mathrm{FB}_{1}, \mathrm{~T}-2$, DON, and ZEN in wheat were 4, 20, 10,200 , and $40 \mu \mathrm{g} / \mathrm{kg}$, respectively. For visual estimation the corresponding cut-off values were 20, 1000, 200, 4000 , and $400 \mu \mathrm{g} / \mathrm{kg}$. Using the reader, the LOD for $\mathrm{AFB}_{1}$ would be acceptable for monitoring in some, but not all, countries. However, for $\mathrm{FB}_{1}, \mathrm{~T}-2$, and $\mathrm{DON}$ the reader provided sensitivity below that required by most regulatory agencies. The limitation of the method appears to be in the sample pre-treatment. Samples were extracted using acetonitrile/water, but to avoid the effects of solvents upon the antibodies, the extracts were centrifuged and dried under nitrogen. The dried extracts were dissolved in aqueous buffer for detection. The need to dry-down the extracts might limit the field-portability of the test, which is unfortunate given the portability of the strips and the reader. Despite this limitation, the incorporation of 5 toxins into one LFIA is helping to move true multiplexed LFIA towards field applications.

Several groups reported the means to improve sensitivity through the use of alternative labels. Quantum dots (QDs) are often substituted for colloidal gold as labels in LFIA, and recently novel fluorescent QDs were developed and tested in a duplex LFIA for DON and ZEN (Goryacheva et al., 2020). The investigators prepared $\mathrm{CdSe} / \mathrm{CdS}$ and CdSe/ $\mathrm{CdS} / \mathrm{ZnS}$ core-shell QDs in various sizes, coated them with $\mathrm{SiO}_{2}$ and then functionalised them with either epoxy or carboxyl groups. DON antibodies were conjugated with redemitting QD-SiO 2 while $\mathrm{ZEN}$ antibodies were conjugated with orange-emitting QD-SiO 2 . The labelled antibodies were then incorporated into a LFIA of the antigenimmobilised format. Responses were observed visually under UV excitation. Cut-off levels for ZEN and DON were adjusted to 40 and $400 \mu \mathrm{g} / \mathrm{kg}$, respectively. Samples of maize (18) and wheat (16) were tested qualitatively by the duplex LFIA and quantitatively by LC-MS/MS, with good agreement between the two methods at the indicated cutoff values. At the cut-offs, sensitivities, determined by the authors as the percentage of samples classified as positive relative to the number of true positive samples, were found to be 96 and $96 \%$ for DON in maize and wheat samples, respectively. Specificities, determined as the percentage of samples classified as negative samples relative to the number of true negative samples, were found to be $97 \%$ for DON in maize and $95 \%$ for DON in wheat. Sensitivities and specificities were each found to be $100 \%$ for ZEN in both maize and wheat. These satisfy the requirements of the European Commission (EC, 2014) for a screening method.

Certain fluorophores will give increased fluorescence when they aggregate. Such aggregation-induced emission luminogens have been used as labels for a duplex LFIA for $\mathrm{AFB}_{1}$ and cyclopiazonic acid (CPA) (Hu et al., 2021). Polystyrene nanospheres containing an aggregationinduced emission-active tetraphenylethylene derivative was synthesised and then conjugated to $\mathrm{AFB}_{1}$ or CPA monoclonal antibodies (mAbs). The mAb conjugates were then incorporated into a traditional duplex competitive LFIA format. Peanut samples were extracted with 70:30 methanol/water $\mathrm{v} / \mathrm{v}$ with added $\mathrm{NaCl}$, filtered, and diluted 3 -fold for testing by LFIA and LC-MS/MS. For AFB 1 the LOD was $0.003 \mathrm{ng} / \mathrm{ml}$, with a linear range between 0.05 and $1.2 \mathrm{ng} / \mathrm{ml}$. For CPA the LOD was $0.01 \mathrm{ng} / \mathrm{ml}$, with a linear range between 0.8 and $50 \mathrm{ng} / \mathrm{ml}$. Based on the extraction and dilution ratios these are equivalent to a linear range between 0.4 to $9.6 \mu \mathrm{g} / \mathrm{kg}$ for $\mathrm{AFB}_{1}$ and 6.4 to $400 \mu \mathrm{g} / \mathrm{kg}$ for CPA. Peanut samples were spiked with $\mathrm{AFB}_{1}$ at three levels $(0.05,5$, and $20 \mu \mathrm{g} / \mathrm{kg})$ and CPA at three levels $(20$, 100 , and $700 \mu \mathrm{g} / \mathrm{kg}$ ). The recoveries ranged from 93 to $110 \%$ for $\mathrm{AFB}_{1}$ and 89 to $98 \%$ for CPA, with RSDs of 3.2 to $5.8 \%$. Strips were stable for 180 days when stored at $4{ }^{\circ} \mathrm{C}$. The assay was applied to 100 samples of peanuts, 12 of which were contaminated with $\mathrm{AFB}_{1}$, seven of which were contaminated with CPA. Five samples were contaminated with both toxins. Good agreement was obtained relative to an LC-MS/MS method, suggesting the aggregation-induced emission luminogens-based LFIA can be used for screening peanuts for these two toxins.

\section{Multiplexed sensor arrays}

Recently a 'hybrid' format incorporating LFIA and microarrays was explored for the assay of five mycotoxins (Charlermroj et al., 2021). The format involved spotting a $7 \times 4$ array, rather than the traditional lines, onto the signal pad of the LFIA device. Four spots were assigned to each of the five toxin-protein conjugates, four were assigned to a positive control, and four were assigned to a negative control (bovine serum albumin, BSA). The presence of toxin was determined by the resulting pattern of spots on the strip, while the concentration was estimated from the (inverse of) the intensity of fluorescence. To facilitate detection a large Stokes-shift organic reporter molecule based on a pentahelicene dye was synthesised and conjugated to a secondary anti-mouse antibody. Detection was based on the formation of a toxin-BSA/ primary antibody/secondary antibody-fluorophore complex excited at $312 \mathrm{~nm}$. The five mycotoxins that were measured were: $\mathrm{AFB}_{1}$, DON, FB 1 , T-2, and ZEN. The LODs were: 1.3, $0.5,0.4,0.4$, and 0.9 'ppb', respectively. Unfortunately, it is unclear what is meant by 'ppb', i.e. whether this refers to the concentration of calibration solutions $(\mu \mathrm{g} / \mathrm{l})$, or whether it refers to concentration in actual solid samples $(\mu \mathrm{g} / \mathrm{kg})$. The difference, of course, is that the latter would incorporate the extraction step and any dilutions of the extract, while the former would not. While recovery data were reported and were good (ranging from 70.7 to $124.8 \%$ ), the details 
of the spiking were not provided, to the extent that even the matrix used for the spiking was not identified. For this reason, it is unclear if the recoveries represent recoveries from spiked extracts or recoveries from spiked samples. Because the article did not report results from specific food or feed samples, it seems likely that 'ppb' referred to $\mu \mathrm{g} / \mathrm{l}$ of standard solutions. Unfortunately, this situation occurs frequently in the literature, and authors are reminded to clearly specify how recovery studies were conducted. In the current article, the impact was likely minor for DON, $\mathrm{FB}_{1}, \mathrm{~T}-2$, and $\mathrm{ZEN}$, as the LODs were well below regulatory levels. However, there may be an impact for $\mathrm{AFB}_{1}$, with a reported LOD of $1.3 \mathrm{ppb}$. If this refers to $1.3 \mu \mathrm{g} / \mathrm{l}$ of standard (or spiked extract), the LOD may not be sufficient to meet European regulations $(E C, 2006)$ when applied to food samples.

Improving the signal obtained from microarrays can be accomplished by a variety of optical and electrochemical means. This includes techniques to increase the number of binding events, and techniques to amplify the reporting of binding events. The former includes modifications to the surfaces of sensors to increase the number of interactions, while the latter includes novel reagents to enhance the detection of such interactions. Recently an approach was used to perform both such functions. A 4-plex microarray was developed wherein the microarray substrate was modified through the deposition of silver onto poly(methyl methacrylate) coated slides that had been treated with oxygen plasma to create 3D micronanostructures (Koukouvinos et al., 2021). The micronanostructures helped to increase surface area, allowing greater antigen binding, while the silver also amplified the fluorescence from the label that was used (AlexaFluor 647; Life technologies, Carlsbad, CA, USA). The array was $4 \times 4$ with toxin-protein conjugates immobilised on the surface. Toxin specific antibodies and sample extracts were incubated for $60 \mathrm{~min}$, followed by addition of a secondary antibody-biotin conjugate (45 min). Visualisation followed incubation with an AlexaFluor-streptavidin reagent $(15 \mathrm{~min})$. The process reportedly took about $2 \mathrm{~h}$, excluding the sample preparation and washing steps, which is long relative many other detection systems. Maize samples were extracted with acetonitrile/water $(80: 20, \mathrm{v} / \mathrm{v})$. The extracts were purified using a QuEChERS mixture, then diluted 1:5 $\mathrm{v} / \mathrm{v}$ for analysis. Results indicated that the nano-microstructuring of the surface and deposition of silver caused a 2.8-fold increase in signal intensity for $\mathrm{AFB}_{1}$ and OTA, a 5.6-fold increase for DON, and a 16fold increase for $\mathrm{FB}_{1}$, without a corresponding increases in non-specific signals. Therefore, the goal of increasing signal intensity through surface modifications was achieved. The linear working ranges for $\mathrm{FB}_{1}, \mathrm{AFB}_{1}$, OTA, and $\mathrm{DON}$ were reportedly $10-400,0.5-20,0.5-400$, and $10-400 \mu \mathrm{g} / \mathrm{kg}$, respectively, with intra-assay RSDs below $7.2 \%$ and interassay RSDs below 9.1\%. As such the assay would cover the ranges needed for application to real samples. Application to naturally-contaminated samples or certified reference materials (CRMs) was not reported.

Another approach to improving the sensitivity of microarrays through modification of the sensor surface was reported by Li et al. (2020). To increase the surface area available for biomolecular interactions, the substrate chosen was a $\mathrm{TiO}_{2}$-modified porous silicon. $\mathrm{The}^{\mathrm{TiO}}{ }_{2}$-modified porous silicon wafers were treated with (3-glycidyloxypropyl) trimethoxysilane to provide epoxide groups to react and immobilise toxin-protein conjugates. Wafers modified with $\mathrm{TiO}_{2}$ resulted in much higher signals than unmodified wafers. Toxins and their associated antibodies were added, washed, and a secondary antibody labelled with the fluorophore Cy3 was used for visualisation. Visualisation was performed by scanning the green fluorescence of the Cy3. The linear detection ranges were reported to be 0.01 to $1 \mathrm{ng} / \mathrm{ml}$ for OTA and $\mathrm{FB}_{1}$, and 0.001 to $1 \mathrm{ng} / \mathrm{ml}$ for $\mathrm{AFB}_{1}$. LODs were calculated as $0.433,0.093$, and $0.243 \mathrm{ng} / \mathrm{ml}$ for $\mathrm{OTA}, \mathrm{FB}_{1}$, and $\mathrm{AFB}_{1}$, respectively. The latter values are confounding, as they place the LODs for OTA and $\mathrm{AFB}_{1}$ near the upper end of their reported linear ranges. Recoveries were obtained from three commodities, maize, wheat, and rice each spiked at three levels (0.01, 0.1, and $1 \mathrm{ng} / \mathrm{g}$ ). Recoveries of $\mathrm{AFB}_{1}$ ranged from 64 to $120 \%$. Recoveries of OTA ranged from 70 to $129 \%$, and recoveries of $\mathrm{FB}_{1}$ ranged from 65 to $124 \%$. The immunoassay included a $1 \mathrm{~h}$ incubation with the antibody/toxin mixture and a $1 \mathrm{~h}$ incubation with the secondary antibody, which indicates that the assay times were likely rather long. However, the report demonstrated how increasing the surface area, by modifying it with $\mathrm{TiO}_{2}$, was able to improve signal, which is often key for improving sensitivity.

Recently a new type of biosensor was developed based upon a form of film bulk acoustic resonance using solid mounted resonators. The technology is similar conceptually to a quartz crystal microbalance, except the resonance occurs in piezoelectric films rather than crystals. Two devices based upon the mass sensitive microarray architecture are manufactured by a Finnish company (Biomensio, Espoo, Finland). A 3-plex immunoassay for T-2, $\mathrm{FB}_{1}$, and ZEN was evaluated using the two devices (Nolan et al., 2021). The two devices differed in the number of microfluidic channels, either one (portable), or four (non-portable). The two devices used the same type of microarray chips, which consisted of a $4 \times 16$ array of sensors. Toxin-protein conjugates were immobilised unto the chip surface, and the binding of toxin-specific antibodies was measured. Simplex and multiplex assays were conducted with toxin standards. For simplex assays using the single channel (portable) device the $\mathrm{IC}_{50}$ sere $1.3,2.0$, and $6.8 \mathrm{ng} / \mathrm{ml}$ for $\mathrm{T}-2, \mathrm{FB}_{1}$, and $\mathrm{ZEN}$, respectively. For multiplex assays using the single channel device, the corresponding $\mathrm{IC}_{50} \mathrm{~s}$ were 6.1, 3.6, and 2.4 ng/ $\mathrm{ml}$, respectively. Sensor chips could be cleaned for re-use 
up to three times. The assays with the single channel device were relatively rapid, circa $10 \mathrm{~min}$, exclusive of extraction and pre-incubation steps. The sensitivities were sufficient to suggest that further testing with foods is warranted, particularly if the technique can be combined with a rapid sample extraction/clean-up procedure. It also seems likely that speed could be improved by finding ways to reduce the pre-incubation which, at $45 \mathrm{~min}$, is much longer than such incubations used in LFIA or in many other biosensors.

In previous years several microbead-based immunoassays have been reported, wherein the identity of the toxin was encoded based upon the colour or fluorescent properties of the beads, with the properties measured by luminometers. Recently a duplex assay based upon microbeads was developed wherein the toxins (OTA or ZEN) were encoded by using different sized beads (Zhang et al., 2021b). The level of toxin was associated with the brightness of the microbeads following the immunoassay. The microbeads were spotted onto a slide and then a cell phone was used to capture an image of the beads, process the image, and use the particle size and brightness values to estimate the concentrations of the two toxins. The immune reaction was competition between toxin-urease conjugates and toxin (sample) for binding to antibody-coated microbeads. The competition incubation was at $37^{\circ} \mathrm{C}$ for $20 \mathrm{~min}$. After washing, urea was added and incubated, then bromocresol purple was added, incubated for $1 \mathrm{~min}$, and a picture was taken with a mobile phone. The image was converted to grey scale, binarized, and used to distinguish the size of the particles and calculate their brightness, which was inversely associated with toxin concentration. The LODs of standard solutions were 0.771 and $1.039 \mathrm{ng} / \mathrm{l}$ for OTA and ZEN, respectively. This was a very promising result. However, close examination reveals high non-specific binding of the ZEN-urease to the OTA antibody and of the OTAurease to the ZEN antibody. The result is that when the two assays were combined the calibration curves had very low slope and large variations in the brightness values. This made it much more difficult to clearly differentiate high and low concentrations of the two toxins. It seems likely that reducing the non-specific binding of the toxin-urease conjugates to their opposing antibodies (i.e. ZEN-urease to OTA mAb), or using other mAbs with less non-specific binding, would improve this. The total analysis time was 30 min and the portability of the technique suggests that it warrants further investigation.

\section{Single mycotoxin, or single mycotoxin family, biosensors}

About 80 papers focusing on single mycotoxin/family immunoassays have been published over the period mid 2020 - mid 2021, if compared to the about 45 published last year, it can be indeed stated that this is a quite dynamic research area.
New emerging technologies have been explored as well as strategies for improving the traditional approaches. However, when a lot of effort is put in immunoreagent synthesis or adaptation of a given technology to mycotoxin detection, less space is left to method testing in real samples. For most of the reviewed cases, it would be interesting to see next year, as a follow up, the application of those innovative approaches in routine analysis in a real-world testing environment. If compared to the detection approaches, less innovation can be found in sample preparation. In spite of the strong trend observed in commercially available products implementing solvent-free extraction procedures, literature methods still stick to the 'traditional' extraction protocols based on methanol/water (or acetonitrile) mixtures.

Having available antibodies paves the way for new assay development. While a wide range of antibodies for regulated mycotoxins is available, research made some steps forward also this year in the development of antibodies for new, unregulated mycotoxins. As an example, the development of $\mathrm{mAbs}$ directed against roquefortine $\mathrm{C}$ (ROC) and their application in a competitive ELISA has been reported by Maragos (2020). ROC is commonly found in foods infested either intentionally or unintentionally with $P$. roqueforti, and has been suggested as candidate biomarker of intoxication by tremorgens, such as penitrem A. Evaluation of cross reactivity towards several compounds with related substructures, showed a very high selectivity of the developed antibody for ROC. The resulting ELISAbased method, requiring no sample pre-treatment other than a 1:5 dilution in buffer, was sensitive for ROC, with a LOD of $0.026 \mathrm{ng} / \mathrm{ml}$, as well as tolerant to significant levels of methanol. Recoveries from 4 types of nut milks spiked over the range of 0.25 to $2 \mathrm{ng} / \mathrm{ml}$ were in the range of 84 to $116 \%$. Applicability of the assay was evaluated by conducting a small survey of commercial nut 'milks' and 'creamers'. Data indicated 4 out of 35 samples contained ROC at levels so low that they were unlikely to be significant to human health $(<0.6 \mathrm{ng} / \mathrm{ml})$. The assay was also applied to canine serum. Recoveries from serum spiked over the range of 0.2 to $5 \mathrm{ng} / \mathrm{ml}$ ranged from 98 to $123 \%$, indicating its potential applicability to the screening of serum from dogs suspected to be suffering from mycotoxin-induced tremors.

Among unregulated mycotoxins, Alternaria toxins have been included in European Commission monitoring programmes since 2012. This official request for generation of occurrence data is driving the development of fit-forpurposes analytical methods. The available data show tenuazonic acid ( $\mathrm{TeA}$ ) to be the most frequently occurring Alternaria toxin and at the highest contamination levels. In the work by Liang et al. (2021) a sandwich ELISA was realised using a pair of matched heavy chain variable region and light chain variable region from hybridoma cells producing an anti-TeA monoclonal antibody. A sandwich 
ELISA detects the antigen (TeA in this case) between two layers of antibodies (capture and detection antibody). The established ELISA was intended for TeA determination in juice, wheat flour, and tomato ketchup. The assay exhibited an LOD of $0.08 \mathrm{ng} / \mathrm{ml}$, and was reported to be 13 times more sensitive than a classical indirect competitive ELISA previously developed by the same group. The improved sensitivity of the assay allowed extract dilution (5, 20 and 50 -fold dilutions) to manage matrix effects. Recoveries from spiked juice, wheat flour and tomato ketchup samples (spiking range from 8 to $50 \mu \mathrm{g} / \mathrm{kg}$ ) ranged from 88 to $111 \%$, with RSDr lower than $15 \%$. A small set of real samples including beer $(n=3)$, wheat flour $(n=4)$, and tomato ketchup $(n=3)$ was also analysed to compare the developed ELISA with a reference UPLC-MS/MS method. For beer and wheat flour samples, consistent results were obtained, however, for tomato ketchup, inconsistency between the two methods was observed. In spite of the sufficient method performances obtained for most of the targeted commodities, it is worth mentioning that TeA extraction from tomato ketchup or wheat flour was achieved by using chloroform, which would significantly limit method application on site or in laboratories with limited analytical equipment or organic solvents waste management procedures.

Fluorescence polarisation immunoassay (FPIA) is a widely used immunoassay, performed in one phase without requiring the immobilisation of reagents. Moreover, it has been recently proven to be suitable for multiplexing. Therefore, FPIA applications to mycotoxin detection continue to deserve attention. Keeping the sample preparation procedure as simple as possible, the achievement of improved sensitivity and selectivity is all about choosing the best tracer/antibody combination. In the study by Huang et al. (2020) different combinations of antibody/tracer have been tested to set up a FPIA for the detection of OTA in rice. OTA-amino-methyl fluorescein resulted to be the best performing tracer. The assay required OTA extraction with $40 \%$ aqueous methanol, followed by dilution 1:4 with water. Analytical performances of the assay (recovery, intra-assay, and inter-assay reproducibility) were evaluated in rice samples spiked with $0.5,5$, and $50 \mu \mathrm{g} /$ $\mathrm{kg}$ OTA. The average recovery range was $70-110 \%$ with a RSDr equal to or less than $10 \%$, thus fulfilling the mycotoxin screening and testing method validation and performance criteria set by the EU (EC, 2014). The trueness of the developed FPIA-based method was assessed by comparison with a reference HPLC-FLD method on a set of 10 rice samples contaminated with OTA at levels ranging from 1 to $15 \mu \mathrm{g} / \mathrm{kg}$, obtaining a satisfactory correlation $\left(\mathrm{r}^{2}=0.9966\right)$.

OTA has been targeted in grape juice by Hao et al. (2020), aiming at demonstrating the suitability of a novel bifunctional magneto-gold nanohybrid (MGNH) as an alternative to conventional gold nanoparticles for colorimetric detection in LFIAs. In complex matrices of a dark colour, such as grape juice, traditional LFIA using AuNPs as signal probes usually suffers from poor detection performance due to a high background colour. Therefore, immunomagnetic separation integrated into the LFIA analysis has been proposed as strategy to minimise the matrix interference via the magnetic-mediated separation and concentration of analytes. Specifically, MGNHs, new magneto-optical bifunctional nanomaterials that simultaneously possess magnetic and colorimetric properties, have been used in this study. Anti-OTA mAbs were coupled with MGNHs to prepare the MGNH-mAb conjugates to be used as competitors in the LFIA. Briefly, grape juice was diluted 1:1 with a buffer, then combined with MGNH-mAb. The formed MGNH-mAb-OTA immunocomplex and free MGNH-mAbs were collected using an external magnetic field and re-suspended in buffer for LFIA. Under optimum conditions, the MGNH-LFIA assay showed a LOD of 0.094 $\mathrm{ng} / \mathrm{ml}$ and average recoveries ranging from 92 to $109 \%$ with a RSDr of below $12 \%$. A comparison between the MGNHLFIA method and a reference LC-MS method measuring 36 OTA-contaminated grape juice samples was carried out obtaining satisfactory correlation $\left(\mathrm{r}^{2}=0.9579\right)$ and no false positive/false negative results.

The use of functionalised magnetic beads in combination with semiconductor QD labelling has been proposed by Xuan et al. (2020) who developed a quantitative electrochemical immunoassay for $\mathrm{AFB}_{1}$ detection, integrating an electrochemical QD-based immunosensor with an automated platform for enrichment, washing, competing, and releasing steps. Samples (maize, wheat, husk rice and peanut oil) were extracted with methanol/water (70:30, v/v). An automated pre-treatment system was set up adopting the immunoaffinity magnetic beads as the capture probe of $\mathrm{AFB}_{1}$ and $\mathrm{QD}$-labelled $\mathrm{AFB}_{1}$ as antigen competitor $\left(\mathrm{AFB}_{1}\right.$-BSA-QDs) as the signal probe. After a washing step, QDs captured on the probe were digested with an acid solution to release corresponding metal ions $\left(\mathrm{Cd}^{2+}\right)$, whose signal was detected by a fast electrochemical detector based on anodic stripping voltammetry. Interesting features of the developed platform are either the automatization of the sample pre-treatment steps and the detection on an unmodified disposable screen-printed electrode that makes the method potentially suitable for replication by other users as well as routine testing. Recovery rates evaluated in spiked samples were between 84 and $118 \%$. The intraday and inter-day RSDr values were in the ranges of $5-18 \%$ and $3-10 \%$, respectively. The lowest spiking levels were comparable with EU maximum limits.

Semiconductor QDs are also used as luminescent labels in biosensors based on Förster resonance energy transfer. This technique is based on the energy transfer from a donor in the excited state to a non-excited acceptor due to dipole-dipole interactions. The Förster resonance energy 
transfer mechanism leads to quenching of donor emission and is strongly dependent on the distance between donor and acceptor. An antigen-antibody interaction enables donor and acceptor to come closer and to realise Förster resonance energy transfer. This approach was explored by Goryacheva et al. (2021) in a Förster resonance energy transfer-based immunoassay for the detection of DON in wheat using DON-ovoalbumin labelled with AuNPs as energy acceptor and anti-DON antibody labelled with green-fluorescent QDs as donor. Synthesis of AuNP and QD labelled immunoreagents and characterisation of the QD-AuNP interaction is reported, as well as the process of setting up the optimal assay condition in buffer solution. When applying the assay on wheat samples (extracted with methanol/water, 70/30, v/v), extract dilution (1:10) was needed due either to the strong influence of the matrix components on the signal or the impact on antibody binding of the high concentration of organic solvent. The calculated LOD for DON in wheat samples was $28 \mu \mathrm{g} / \mathrm{kg}$. No extensive method testing was performed with wheat samples, therefore no data on method analytical performances are reported to enable its comparison with other immunoassays.

Another example of application of QDs for improved detection in LFIA has been reported by $\mathrm{Wu}$ et al. (2021). The aim was to improve the detection of $\mathrm{AFB}_{1}$ in botanical materials by colloidal gold based LFIA, which is hampered by strong matrix effects. For this purpose the authors synthesised a QD-labelled anti-AFB ${ }_{1}$ antibody to be embedded in the sample pad of the strip membrane. The antibody was selected in order to manufacture a strip test capable to detect the sum of $A F B_{1}, A_{2 F}$, aflatoxin $\mathrm{G}_{1}\left(\mathrm{AFG}_{1}\right)$, aflatoxin $\mathrm{G}_{2}\left(\mathrm{AFG}_{2}\right), \mathrm{AFM}_{1}$, and aflatoxin $\mathrm{M}_{2}$ $\left(\mathrm{AFM}_{2}\right)$. For method development a mix of 10 botanical medicines was prepared and spiked with the target aflatoxins in the range $2-200 \mu \mathrm{g} / \mathrm{kg}$. Sample were extracted with methanol/water $(70: 30, \mathrm{v} / \mathrm{v})$ then diluted $1: 7$ to be applied to the strip test. Matrix-matched calibration curves were used for quantitative analysis. Estimated LODs for individual aflatoxins ranged 1 to $2 \mu \mathrm{g} / \mathrm{kg}$. To demonstrate potential applicability and usefulness in the analysis of real samples, a set of 50 botanical medicine samples were analysed by the developed QD-LFIA strip and the Chinese national standard HPLC method as a reference. Aflatoxins were detected in $22 / 50$ samples at levels up to $16 \mu \mathrm{g} / \mathrm{kg}$. Interestingly, the paper also reports on the development of a strip test reader, with relevant software. The QD reader mainly consisted of the optical system to generate excitation light, the photoelectric conversion system to convert the optical signal to a voltage signal, and the high-performance microprocessor for signal processing and data calculation. The software system operating under Windows XP or higher versions of Windows, provided main functions of processing QD-LFIA strip images, analysis, data storage, and results printing.
Besides botanical medicines, animal-derived medicines is another important part of traditional Chinese medicine, that was indicated as a target for $\mathrm{AFB}_{1}$ determination by Wang et al. (2021). The contamination level of AFs in animal medicines is reported to be significantly higher than the average level of other traditional Chinese medicines, so the authors proposed the application of an ELISA method for risk assessment purposes. Animal-derived medicines considered in this study were ground beetle, cockroach, silkworm, and earthworm. The sample powder was extracted with acetonitrile, then dried down and resuspended in phosphate buffer to make it compatible with the ELISA test. A traditional indirect competitive ELISA was set up, showing sufficient analytical performances to be applied to the analysis of a total of 138 animal material samples, collected from medicinal markets and stores, across four different provinces. Based on the detected $\mathrm{AFB}_{1}$ contamination, quantitative risk assessment was performed by calculating the population cancer risk caused by animalderived medicines intake using the approach established by JECFA. This approach enables the estimation of the number of liver cancer cases per 100,000 individuals per year. Data showed that the population cancer risk related to the consumption of ground beetle (risk of liver cancer of 0.036 cancers per 100,000 individuals) exceeded the tolerable risk reference values of carcinogens calculated according to the European Chemicals Agency (ECA, 2012).

A further example of rapid immunoassay-based test application for human exposure assessment can be found in the literature this year. Wang et al. (2020) developed a surface-enhanced Raman spectroscopy (SERS)-based lateral flow immunosensor for rapid monitoring of $\mathrm{AFM}_{1}$, the $\mathrm{AFB}_{1}$ biomarker of exposure, in urine. To this purpose, the Raman molecule 5,5-dithiobis-2-nitrobenzoic acid and an anti-AFM 1 monoclonal antibody were conjugated with AuNPs to serve as the SERS nanoprobe. A microwell lateral flow format was employed for the competitive

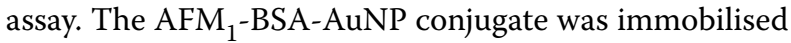
on the test line of nitrocellulose membrane as the capture reagent, while the anti-AFM ${ }_{1}$ SERS nanoprobe was dried in a micro-plate well. Quantification of $\mathrm{AFM}_{1}$ was performed by the readout of Raman signal from the SERS nanoprobes captured on the test line. Sample pre-treatment required only urine centrifugation followed by dilution with buffer. The assay detection limit was about $2 \mathrm{pg} / \mathrm{ml}$ of $\mathrm{AFM}_{1}$ in urine, whereas spiking experiments yielded 94-111\% recovery with coefficients of variation below $17 \%$. Overall, the method performances indicated its suitability for rapid aflatoxin biomonitoring in human urine.

Among selected research works it is finally worth to include an example of smartphone-based biosensor. Indeed, smartphones represent an ideal device for onsite/ online detection, as they combine pervasive distribution with rapidly developing technologies for connectivity, 
customisable applications, image acquisition and processing. Integration with paper-based assay technology is already a reality and some platforms are available on the market. A fluidic integrated system was proposed by Zangheri $e t$ al. (2021). It consisted of a smartphone integrated selfstanding device comprising a low-cost and disposable analytical cartridge containing all the reagents required for the execution of the analysis. OTA detection on the strip test membrane was based on chemiluminescence. The smartphone CMOS (complementary metal-oxide semiconductor) camera was used as a light detector. Chemiluminescence images were then analysed by the freely available image analysis software ImageJ. Target matrices were red and white wine, as well as instant coffee (dissolved in water), that were mixed with aqueous solutions of $\mathrm{NaHCO}_{3} / \mathrm{PEG} 10000$ to remove substances that could interfere with the chemiluminescence detection (e.g. polyphenols). Matrix-matched calibration curves were used for quantitative analysis. Recoveries of the optimised assay ranged from 81 to $123 \%$ with RSDr lower than $15 \%$, whereas LODs ranged from 0.3 and $0.1 \mu \mathrm{g} / \mathrm{l}$, respectively, which are below the European regulations. The shelf life of the analytical cartridges at $4{ }^{\circ} \mathrm{C}$ was estimated to be about one year, however no data regarding the maximum number of samples that could be analysed with the same cartridge were reported. Indeed, this is a critical factor in fluidic systems, that may significantly affect analysis costs in routine use.

\section{Assays with antibody analogues}

Although immunoaffinity-based separations and assays remain the touchstone for mycotoxin analysis, certain practical limitations with antibody technology, such as batch to batch variation, limited shelf life, and cold chain requirements, have continued to motivate the search for alternative approaches. Competing approaches based on synthetic receptors such as aptamers and molecularly imprinted polymers (MIPs) remain of great interest. Aptamers are biopolymers (typically singlestranded oligonucleotides) that are discovered through a combinatorial selection process for their affinity and specificity for a target of interest. MIPs, also known as 'plastic antibodies', are synthetic polymers that are synthesised in the presence of a molecular template that leaves the materials with a pre-determined selectivity for a particular analyte.

Given the familiarity and acceptance of conventional antibody-based detection systems, aptamer and MIP-based strategies must find their niche in mycotoxin analysis by profiting from their practical advantages, in particular their relatively low cost of production and their extended shelf-lives. In particular, alternative synthetic receptors could have a marked advantage in the development of inexpensive screening technology for on-site testing applications that require extensive sampling. Several reports over the past year have focused on exploiting some of these advantages, but unfortunately many others, while inventive and attractive from an academic standpoint, are unlikely to be practical for field use.

A common strategy in alternative mycotoxin detection methods is to simply replace the antibody in an established assay with a MIP or aptamer affinity agent. For example, ELISA is a staple detection method for mycotoxin analysis. Equivalent enzyme-linked apta-sorbent assays (ELASA) have been developed in recent years in the hopes of lowering costs and improving shelf-life by replacing the capture and/or detection antibody with an equivalent aptamer. Recently, Mukherjee and co-workers (2021) developed colorimetric and chemiluminescent ELASAs for OTA monitoring in food. In each assay, one of the two antibodies in the sandwich format was replaced with an aptamer and compared to a traditional sandwich ELISA; both ELASAs were comparable in sensitivity with a broader detection range than the ELISA format when challenged under optimised buffer conditions. Limits of detection for each format in spiked solutions were in the $1 \mathrm{pg} / \mathrm{ml}$ range; determination of the sensitivity in sample matrix was not described. Recovery studies from coffee beans and groundnuts spiked at the $1 \mu \mathrm{g} / \mathrm{kg}$ level showed acceptable recoveries, with the best performance from the chemiluminescent ELASA platform (91-108\% recovery).

Electrochemical mycotoxin biosensors have potential for real world application thanks to their advantages such as low cost and potential for miniaturisation and portability. MIP technology is especially attractive for use with electrochemical detection because the MIP can be prepared directly on the electrode surface by electropolymerization methods. Hatamluyi et al. (2020) prepared a MIP-based patulin biosensor by electropolymerization atop a glassy carbon electrode that had first been decorated with graphene QDs and a gold nanoparticle-functionalised metal-organic framework (Au@Cu-MOF). Changes in the peak current of the Au@Cu-MOF could be observed after only $180 \mathrm{~s}$ of PAT incubation with the MIP sensor. The sensor presented a linear range of $0.001-70.0 \mathrm{ng} / \mathrm{ml}$, a low detection limit of $0.0007 \mathrm{ng} / \mathrm{ml}$, and good selectivity for PAT over other targets such as 5-hydroxymethyl-furfural, $\mathrm{AOH}$, $\mathrm{OTA}$, and $\mathrm{AFB}_{1}$. Recoveries from spiked apple juice samples (from $0.005 \mathrm{ng} / \mathrm{ml}$ to $50 \mathrm{ng} / \mathrm{ml}$ ) ranged from 98 to $99 \%$ with RSD values from 1.2 to $4.6 \%$. Finally, sensor performance on apple juice concentrate samples was compared to HPLC using AOAC Official Method 995.10 and there was no statistically significant difference in the findings. Overall, the approach seems promising but could face barriers to translation because of the unconventional components in the electrode material. 
In a similar example, Munawar et al. (2020) developed a MIP-based sensor for $\mathrm{FB}_{1}$ by first preparing a nanoMIPs template with $\mathrm{FB}_{1}$ and then depositing those nanoparticles onto a coated platinum electrode. The LOD from simple buffer solutions was determined to be $0.03 \mathrm{fM}$ using electrochemical impedence spectroscopy and $0.7 \mathrm{fM}$ using differential pulse voltammetry. $\mathrm{FB}_{1}$ recovery from maize (samples from 8.3 to $62.5 \mu \mathrm{g} / \mathrm{kg}$ ) showed recoveries of 96 to $101 \%$. This approach is more straightforward than the one described by Hatamluyi et al. (2020) and the possibility to drop the cost of the assay by using disposable screen printed platinum electrodes and a handheld potentiostat system would be worth exploring.

Aptamers are also well-suited for electrochemical biosensing based on the ease with which they can assemble into monolayers on the electrode surface by chemisorption or covalent attachment. For example, Rahimi et al. (2021) developed an electrochemical aptasensor for detection of $\mathrm{AFB}_{1}$ by the chemisorption of an amine-modified $\mathrm{AFB}_{1}$ aptamer onto a glassy carbon electrode modified with a carbon dot $/ \mathrm{Cu}_{2} \mathrm{O}$ nanocomposite. The 'signal-off' biosensor showed a loss of redox signal as $\mathrm{AFB}_{1}$ concentration increased with a linear range of $3 \mathrm{ag} / \mathrm{ml}$ to $1.9 \mu \mathrm{g} / \mathrm{ml}$ and a calculated LOD ( $3 \times$ standard deviation of the blank/ slope of the calibration curve) of $0.9 \pm 0.04 \mathrm{ag} / \mathrm{ml}$. Recovery experiments from wheat samples fortified at the micromolar level $(0.78$ to $2.3 \mathrm{mg} / \mathrm{kg})$ showed recoveries from 97 to $104 \%$ with RSD values from $2.4-3 \%$. In this case, while the carbon electrode and nanocomposite are all relatively low-cost components, the complexity of the design may restrict its implementation.

Gökçe et al. (2020) developed an aptamer-based electrochemical sensor with disposable pencil graphite electrodes by first electrografting 4-carboxyphenyl to the electrode surface and then covalently immobilising aminemodified OTA aptamers via amide coupling chemistry. This simple biosensor assay used impedimetric detection for OTA measurements in buffer with a LOD of $0.1 \mathrm{ng} / \mathrm{ml}$. Spiked beer samples (0.4-1.6 ng/ml) were also tested using this biosensor with recoveries from 92 to $94 \%$ and RSD values of 3.5 to $5 \%$. Comparison of these results to those of similar electrochemical assays using the same aptamer but on screen printed carbon electrodes suggests comparable analytical performances at greatly reduced fabrication costs.

Another sensor platform that is very compatible with antibody analogue technology is based on surface plasmon resonance (SPR). SPR sensors have gained popularity in recent years for their ability to perform label-free, real-time measurements and the recent development of benchtop or portable SPR systems could allow for more widespread use in mycotoxin testing. Just as with electrochemical sensing, MIP technology is well-suited for SPR assays as the MIP can be polymerised directly on the SPR chip surface. Recently,
Akgönüllü et al. (2021) prepared a MIP SPR sensor for OTA detection by photo-polymerising the OTA-imprinted polymer film on the sensor chip surface. Using aqueous OTA solutions, a LOD of $0.028 \mathrm{ng} / \mathrm{ml}$ was calculated. Recoveries from spiked dried fig samples were tested in the range of 1.6 to $32 \mu \mathrm{g} / \mathrm{kg}$ both by this method and by HPLC. Recoveries from 98 to $100 \%$ with RSD values from 1.8 to $2.5 \%$ were observed with SPR; similar values were found when the samples were analysed by HPLC (90 to $100 \%$ recovery with RSD values of 1.7 to $3.5 \%)$. A second study by Akgönüllü et al. (2020) used a more complex MIP approach to detect $\mathrm{AFB}_{1}$ by SPR. The MIP coating was once again prepared by photo-polymerisation on the chip surface, however, gold nanoparticles were also embedded within the film to increase surface area and improve sensitivity. In this case, the calculated LOD from buffer solutions was $1 \mathrm{pg} / \mathrm{ml}$. Recoveries from spiked maize and peanut sample extract solutions (1-10 ng/ml) ranged between 97 and 106\%, which was comparable to what was found when the same samples were analysed by HPLC (87-106\% recovery).

Aptamer-based sensing approaches can take advantage of their unique nature as oligonucleotides to conceive of new assays designs that would not be possible with antibody affinity agents. For example, aptamer assays can capitalise on the ability of DNA to assemble in a predictable and programmable way based on complementary base-pair hybridisation. With this in mind, Xiong et al. (2021) developed a one-step fluorescence assay for detection of $\mathrm{AFB}_{1}$ and OTA called a DNA 'tweezers' nanomachine. A DNA assembly comprised of aptamers and fluorophorelabelled complementary sequences were assembled into a nanoscale structure that keeps two fluorophores in close proximity to their respective quencher molecules (tweezers closed). In this locked conformation, there is no fluorescence detectable. In the presence of one or both of the toxins, however, the aptamers release their complementary sequences in order to bind their cognate target. This structural change (tweezers open) allows the fluorophores to be spatially separated from their quencher, leading to an increase in fluorescence signal. The target can be identified by what fluorescence signal is increased and the intensity is proportional to its concentration. Linear relationships were observed between the fluorescent intensities and $\mathrm{AFB}_{1}$ and OTA concentrations from 0.08 to $10 \mu \mathrm{g} / \mathrm{kg}$ for $\mathrm{AFB}_{1}$ and 0.5 to $50 \mu \mathrm{g} / \mathrm{kg}$ for OTA. The LODs in simple aqueous solution were found to be $0.035 \mu \mathrm{g} / \mathrm{kg}$ for $\mathrm{AFB}_{1}$ and $0.1 \mu \mathrm{g} / \mathrm{kg}$ for OTA, respectively. This solutionbased assay was used to analyse spiked food samples, such as maize, peanut, coffee, olive oil, and peanut oil. Recoveries between 95 and 106\% with RSD values of 4.1-8.1\% were reported. Comparable values were obtained when the same samples were analysed by HPLC. Assays capable of simultaneous, low-cost screening of multiple mycotoxins could also be a niche for synthetic antibody analogues. 
Another unique advantage of aptamer technology in particular, is that methods for the direct exponential amplification of oligonucleotides can be exploited to dramatically improve the sensitivity of the designed assay in ways that would not be possible with an antibody recognition element. For example, Santovito et al. (2020) recently designed a magnetic bead-based sandwich-assay for OTA with two phases of signal amplification. Firstly, binding of OTA to a capture aptamer triggers an isothermal rolling circle amplification (RCA) reaction. After $4 \mathrm{~h}$ of incubation, the DNA amplification is so extensive that a DNA precipitate is visible in the sample. Secondly, the RCA product is a DNAzyme (catalytic DNA sequence) with peroxidase activity, capable of producing a blue-colour by oxidising ABTS [2,2'-azino-bis(3-ethylbenzothiazoline-6sulfonic acid)] substrate that is visible by the naked eye and quantifiable by UV-vis spectroscopy. The authors posited that this test could be used to detect OTA in biological fluids for biomonitoring and exposure assessments, thus they focused on urine as the matrix of interest. As a result of the considerable signal amplification, the authors claim that the LOD in simple buffer solution was $1 \times 10^{-12} \mathrm{ng} / \mathrm{ml}$ ( $<2$ OTA molecules $/ \mathrm{ml}$ ). Recovery experiments from spiked rat urine using more substantial OTA quantities $(0.001$, $0.01,0.1,1$, and $10 \mathrm{ng} / \mathrm{ml}$ ) yielded satisfactory overall average recoveries (>95\%), with RSDs in the range 3.6-15\%. Furthermore, naturally contaminated urine from rats fed OTA contaminated feed was analysed both by this assay and by HPLC-FLD with excellent agreement.

Niazi et al. (2020) also used RCA to prepare a fluorescencebased aptasensor for $\mathrm{AFM}_{1}$ detection in milk. In this design, the presence of the target prevented the aptamer from forming a complex with a rolling circle template, thus inhibiting the production of the RCA product and leading to a lower fluorescence signal generated by a $\mathrm{Eu}^{3+}$ fluorescent nanoparticle. The multi-step process required $3 \mathrm{~h}$ of incubation time but was able to achieve an LOD of $0.0194 \mathrm{pg} / \mathrm{ml}$ in simple buffer solution, with good specificity against $\mathrm{AFB}_{1}, \mathrm{AFB}_{2}, \mathrm{~T}-2, \mathrm{FB}_{1}$ and $\mathrm{ZEN}$. The recoveries of $\mathrm{AFM}_{1}$ in milk (0.001, 0.05, 0.1 and $1 \mathrm{ng} /$ $\mathrm{ml}$ ) were between 92 and 100\% with RSD values less than $5 \%$. Comparison of the assay to a standard ELISA showed high correlation. While the extended incubation time and the multiple steps are limitations of these RCA methods, this approach could be adapted for point-of-use mycotoxin screening in food and feed given that RCA is isothermal and obviates the need for thermal cycling equipment. Both the colorimetric and fluorometric approach, however, would require portable spectrophotometers or fluorimeters, although these are becoming more affordable and commonplace.

Studies highlighted here were chosen for having some degree of potential applicability to in-field testing. A major barrier to the translation of these new approaches to real world mycotoxin screening is the lack of analysis of reference materials, comparison with reference methods, and/or interlaboratory studies to verify method reliability. Often, these studies with antibody analogues limit their testing to dilution series of aqueous solutions, or occasionally recovery experiments from a spiked sample. Another major impediment to the widespread adoption of aptamer and MIP technology to mycotoxin analysis is the trend towards more complex and costly biosensor and assay platforms without consideration of the impracticality of these approaches in real-world scenarios. While these studies often boast low detection limits, appreciable sensitivity, and innovative designs, the need for exotic components, multi-step preparation, and complex instrumentation negate the advantages that aptamer and MIP technology can offer. The field would be well-served by a renewed focus on portable, stable, low-cost approaches that are compatible with point-ofuse testing in order to maximise the benefit of antibody analogues for mycotoxin testing.

\section{Acknowledgements}

Mention of trade names or commercial products in this publication is solely for the purpose of providing specific information and does not imply recommendation or endorsement by the U.S. Department of Agriculture. This work was in part supported by the U.S. Department of Agriculture, Agricultural Research Service. The U.S. Department of Agriculture is an equal opportunity provider and employer. Milena Stranska was financially supported by project GACR 20-14649S.

\section{Conflict of interest}

The authors declare no conflict of interest.

\section{References}

Akgönüllü, S., Armutcu, C. and Denizli, A., 2021. Molecularly imprinted polymer film based plasmonic sensors for detection of ochratoxin A in dried fig. Polymer Bulletin 2021. https://doi. org/10.1007/s00289-021-03699-6

Akgönüllü, S., Yavuz, H. and Denizli, A., 2020. SPR nanosensor based on molecularly imprinted polymer film with gold nanoparticles for sensitive detection of aflatoxin $B_{1}$. Talanta 219: 121219. https://doi. org/10.1016/j.talanta.2020.121219

Belajová, E., 2020. Optimization and applicability of high performance liquid chromatographic methods for quantification of patulin and ochratoxin A in fruit purées. Journal of Food and Nutrition Research 59: 290-300.

Braun, D., Eiser, M., Puntscher, H., Marko, D., and Warth, B., 2021. Natural contaminants in infant food: the case of regulated and emerging mycotoxins. Food Control 123: 107676. https://doi. org/10.1016/j.foodcont.2020.107676 
Çetinkaya, M.A. and Çetinkaya, G., 2021. The mycotoxin proficiency testing schemes organized by Turkish National Food Reference Laboratory between the years 2013 and 2020. Accreditation and Quality Assurance 26: 61-68. https://doi.org/10.1007/s00769-02101463-x

Charlermroj, R., Phuengwas, S., Makornwattana, M., Sooksimuang, T., Sahasithiwat, S., Panchan, W., Sukbangnop, W., Elliott, C.T. and Karoonuthaisiri, N., 2021. Development of a microarray lateral flow strip test using a luminescent organic compound for multiplex detection of five mycotoxins. Talanta 233: 122540. https://doi. org/10.1016/j.talanta.2021.122540

Chavez, R.A., Cheng, X., Herrman, T.J. and Stasiewicz, M.J., 2022. Single kernel aflatoxin and fumonisin contamination distribution and spectral classification in commercial corn. Food Control 131: 108393. https://doi.org/10.1016/j.foodcont.2021.108393

Cheng, X., Chavez, R.A. and Stasiewicz, M.J., 2020. When to use one-dimensional, two-dimensional, and Shifted Transversal Design pooling in mycotoxin screening. PLoS ONE 15: e0236668. https:// doi.org/10.1371/journal.pone.0236668

De Girolamo, A., Ciasca, B., Pascale, M., and Lattanzio, V. M. T., 2020. Determination of zearalenone and trichothecenes, including deoxynivalenol and its acetylated derivatives, nivalenol, T-2 and HT-2 toxins, in wheat and wheat products by LC-MS/MS: a collaborative study. Toxins 12: 786. https://doi.org/10.3390/ toxins 12120786

European Chemicals Agency (ECA), 2012. Guidance on information requirements and chemical safety assessment. Chapter R.8: Characterisation of dose [concentration]-response for human health. Version 2.1 November 2012. Available at: https://tinyurl. com/2p9efkvt.

European Commission (EC), 2006. Commission Regulation (EU) No 4001/2006 of 23 February 2006 laying down the methods of sampling and analysis for the official control of the levels of mycotoxins in foodstuffs. Official Journal of the European Union L 70: 12-34.

European Commission (EC), 2013. Mandate for standardisation addressed to CEN for methods of analysis for mycotoxins in food. Available at: https://law.resource.org/pub/eu/mandates/m520.pdf European Commission (EC), 2014. Commission Regulation (EU) No 519/2014, of 16 May 2014, amending Regulation (EC) No $401 / 2006$ as regards methods of sampling of large lots, spices and food supplements, performance criteria for T-2, HT-2 toxin and citrinin and screening methods of analysis. Official Journal of the European Union L 147: 29-43.

European Commission (EC), 2020. Chemicals strategy for sustainability towards a toxic-free environment, Progress report on the assessment and management of combined exposures to multiple chemicals (chemical mixtures) and associated risks. Available at: https:// ec.europa.eu/environment/pdf/chemicals/2020/10/SWD_mixtures. pdf

European Commission (EC), 2021. Commission Regulation (EU) No 2021/1399 of 24 August 2021 amending Regulation (EC) No $1881 / 2006$ as regards maximum levels of ergot sclerotia and ergot alkaloids in certain foodstuffs. Official Journal of the European Union L 301: 1-5.
European Committee for Standardization, 2019. EN17280:2019 Foodstuffs - Determination of zearalenone and trichothecenes including deoxynivalenol and its acetylated derivatives (3-acetyldeoxynivalenol and 15-acetyl-deoxynivalenol), nivalenol, T-2 toxin and HT-2 toxin in cereals and cereal products by LC-MS/MS. European Committee for Standardization: Brussels, Belgium.

Gbashi, S., Njobeh, P.B., Madala, N.E., De Boevre, M., Kagot, V. and De Saeger, S., 2020. Parallel validation of a green-solvent extraction method and quantitative estimation of multi-mycotoxins in staple cereals using LC-MS/MS. Scientific Reports 10: 10334. https://doi. org/10.1038/s41598-020-66787-z

Goessens, T., Baere, S., Troyer, N., Deknock, A., Goethals, P., Lens, L., Pasmans, F. and Croubels, S., 2021. Multi-residue analysis of 20 mycotoxins including major metabolites and emerging mycotoxins in freshwater using UHPLC-MS/MS and application to freshwater ponds in flanders, Belgium. Environmental Research 196: 110366. https://doi.org/10.1016/j.envres.2020.110366

Gökçe, G., Aissa, S. B., Nemčeková, K., Catanante, G., Raouafi, N. and Marty, J.-L., 2020. Aptamer-modified pencil graphite electrodes for the impedimetric determination of ochratoxin A. Food Control 115: 107271. https://doi.org/10.1016/j.foodcont.2020.107271

Gonçalves, C., Mischke, C. and Stroka, J., 2020. Determination of deoxynivalenol and its major conjugates in cereals using an organic solvent-free extraction and IAC cleanup coupled in-line with HPLCPCD-FLD. Food Additives and Contaminants Part A 37: 1765-1776. https://doi.org/10.1080/19440049.2020.1800829

González-Jartín, J.M., Rodríguez-Cañás, I., Alfonso, A., Sainz, M.J., Vieytes, M.R., Gomes, A., Ramos, I. and Botana, L.M., 2021. Multianalyte method for the determination of regulated, emerging and modified mycotoxins in milk: QuEChERS extraction followed by UHPLC-MS/MS analysis. Food Chemistry 356: 129647. https:// doi.org/10.1016/j.foodchem.2021.129647

Goryacheva, O.A., Beloglazova, N.V., Goryacheva, I.Y. and De Saeger, S., 2021. Homogenous FRET-based fluorescent immunoassay for deoxynivalenol detection by controlling the distance of donoracceptor couple. Talanta 225: 121973. https://doi.org/10.1016/j. talanta.2020.121973

Goryacheva, O.A., Guhrenz, C., Schneider, K., Beloglazova, N.V., Goryacheva, I.Y., De Saeger, S. and Gaponik, N., 2020. Silanized luminescent quantum dots for the simultaneous multicolor lateral flow immunoassay of two mycotoxins. ACS Applied Materials and Interfaces 12: 24575-24584. https://doi.org/10.1021/acsami.0c05099 Guo, W., Yang, J., Niu, X., Tangni, E.K., Zhao, Z. and Han, Z., 2021. A reliable and accurate UHPLC-MS/MS method for screening of: Aspergillus, Penicillium and Alternaria mycotoxins in orange, grape and apple juices. Analytical Methods 13: 192-201 https://doi. org/10.1039/D0AY01787F

Hao, L., Chen, J., Chen, X., Tongtong, M., Cai, X., Duan, H., Leng, Y., Huang, X. and Xiong, Y., 2020. A novel magneto-gold nanohybridenhanced lateral flow immunoassay for ultrasensitive and rapid detection of ochratoxin A in grape juice. Food Chemistry 336: 127710. https://doi.org/10.1016/j.foodchem.2020.127710 
Hatamluyi, B., Rezayi, M., Beheshti, H.R. and Boroushaki, M.T., 2020. Ultra-sensitive molecularly imprinted electrochemical sensor for patulin detection based on a novel assembling strategy using Au@ Cu-MOF/N-GQDs. Sensors and Actuators B: Chemical 318: 128219. https://doi.org/10.1016/j.snb.2020.128219

Hu, X., Zhang, P., Wang, D., Jiang, J., Chen, X., Liu, Y., Zhang, Z., Tang, B. and Li, P., 2021. AIEgens enabled ultrasensitive point-of-care test for multiple targets of food safety: aflatoxin $B_{1}$ and cyclopiazonic acid as an example. Biosensors and Bioelectronics 182: 113188. https://doi.org/10.1016/j.bios.2021.113188

Huang, X., Tang, X., Jallow, A., Qi, X., Zhang, W., Jiang, J., Li, H., Zhang, Q. and Li. P., 2020. Development of an ultrasensitive and rapid fluorescence polarization immunoassay for ochratoxin A in rice. Toxins 12: 682. https://doi.org/10.3390/toxins12110682

International Standards Organization (ISO), 2015. ISO 13528 Statistical methods for use in proficiency testing by interlaboratory comparison, $2^{\text {nd }}$ ed. International Standards Organization, Geneva, Switzerland.

Kerry, R., Ingram, B.R., Garcia-Cela, E. and Magan, N., 2021. Investigation of the potential to reduce waste through sampling and spatial analysis of grain bulks. Biosystems Engineering 207: 92-105. https://doi.org/10.1016/j.biosystemseng.2021.05.001

Koukouvinos, G., Karachaliou, C.-E., Kanioura, A., Tsougeni, K., Livaniou, E., Kakabakos, S.E. and Petrou, P.S., 2021. Fluorescence enhancement on silver-plated plasma micro-nanostructured 3D polymeric microarray substrates for multiplex mycotoxin detection. Processes 9: 392-392. https://doi.org/10.3390/pr9020392

Kumar, A., Dhanshetty, M. and Banerjee, K., 2020. Development and validation of a method for direct analysis of aflatoxins in animal feeds by ultra-high-performance liquid chromatography with fluorescence detection. Journal of AOAC International 103: 940-945. https://doi. org/10.1093/jaoacint/qsz037

Kumphanda, J., Matumba, L., Monjerezi, M., Whitaker, T.B., De Saeger, S. and Makun, H.A., 2021. Are sample size and sample preparation for mycotoxin quantitation in grain products getting trivialized? Food Control 130: 108400. https://doi.org/10.1016/j. foodcont.2021.108400

Laposha, O., Senin, S., Midyk, S., Iakubchak, O., Taran, T., Zabarna, I., Ishchenko, L., Ishchenko, V., and Ushkalov, V., 2020. Determination of T-2 and HT-2 toxin in wheat grain by HPLC with fluorescence detection. Methods and Objects of Chemical Analysis 15: 137-143. https://doi.org/10.17721/moca.2020.137-143

Li, J., Cai, T., Li, W., Li, W., Song, L., Li, Q., Lv, G., Sun, J., Jiao, S., Wang, S., Jin, Y. and Zheng, T., 2020. Highly sensitive simultaneous detection of multiple mycotoxins using a protein microarray on a TiO2-modified porous silicon surface. Journal of Agricultural and Food Chemistry 69: 528-536. https://doi.org/10.1021/acs. jafc.0c06859

Liang, Y., Wang, Y., Wang, F., Li. J., Wang, C., Dong, J., Ueda, H., Xiao, Z., Shen, Y., Xu, Z. and Wang, H., 2021. An enhanced open sandwich immunoassay by molecular evolution for noncompetitive detection of Alternaria mycotoxin tenuazonic acid. Food Chemistry 361: 130103. https://doi.org/10.1016/j.foodchem.2021.130103
Limay-Rios, V. and Schaafsma, A.W., 2021. Relationship between mycotoxin content in winter wheat grain and aspirated dust collected during harvest and after storage. ACS Omega 6: 18571871. https://doi.org/10.1021/acsomega.0c04256

Luci, G., 2020. A rapid HPLC-FLD method for ochratoxin detection in pig muscle, kidney, liver by using enzymatic digestion with MISPE extraction. MethodsX 7: 100873. https://doi.org/10.1016/j. mex.2020.100873

Maragos, C.M., 2020. Development and characterisation of a monoclonal antibody to detect the mycotoxin roquefortine $\mathrm{C}$. Food Additives and Contaminants Part A 37: 1777-1790. https:// doi.org/10.1080/19440049.2020.1781937

Mukherjee, M., Nandhini, C. and Bhatt, P., 2021. Colorimetric and chemiluminescence based enzyme linked apta-sorbent assay (ELASA) for ochratoxin A detection. Spectrochimica Acta Part A: Molecular and Biomolecular Spectroscopy 244: 118875. https:// doi.org/10.1016/j.saa.2020.118875

Munawar, H., Garcia-Cruz, A., Majewska, M., Karim, K., Kutner, W. and Piletsky, S. A., 2020. Electrochemical determination of fumonisin $B_{1}$ using a chemosensor with a recognition unit comprising molecularly imprinted polymer nanoparticles. Sensors and Actuators B: Chemical 321: 128552. https://doi.org/10.1016/j. snb.2020.128552

Narváez, A., Izzo, L., Rodríguez-Carrasco, Y. and Ritieni, A., 2021. Citrinin dietary exposure assessment approach through human biomonitoring high-resolution mass spectrometry-based data. Journal of Agricultural and Food Chemistry 69: 6330-6338. https:// doi.org/10.1021/acs.jafc.1c01776

Ndaw, S., Jargot, D., Antoine, G., Denis, F., Melin, S. and Robert, A., 2021. Investigating multi-mycotoxin exposure in occupational settings: A biomonitoring and airborne measurement approach. Toxins 13: 54. https://doi.org/10.3390/toxins13010054

Niazi, S., Khan, I. M., Yu, Y., Pasha, I., Lv, Y., Mohsin, A., Mushtaq, B. S. and Wang, Z. 2020. A novel fluorescent aptasensor for aflatoxin $\mathrm{M}_{1}$ detection using rolling circle amplification and g-C3N4 as fluorescence quencher. Sensors and Actuators B: Chemical 315: 128049. https://doi.org/10.1016/j.snb.2020.128049

Nolan, P., Auer, S., Spehar, A., Oplatowska-Stachowiak, M. and Campbell, K., 2021. Evaluation of mass sensitive micro-array biosensors for their feasibility in multiplex detection of low molecular weight toxins using mycotoxins as model compounds. Talanta 222: 121521. https://doi.org/10.1016/j.talanta.2020.121521

Pernica, M., Martiník, J., Boško, R., Zuštáková, V., Benešová, K. and Běláková, S., 2021. Determination of patulin and hydroxymethylfurfural in beverages by UPLC-PDA. World Mycotoxin Journal 14: 41-48. https://doi.org/10.3920/wmj2020.2587 Peters, J., Ash, E., Gerssen, A., Van Dam, R., Franssen, M.C.R. and Nielen, M.W.F., 2021. Controlled production of zearalenoneglucopyranoside standards with Cunninghamella strains using sulphate-depleted media. Toxins 13: 366. https://doi.org/10.3390/ toxins13060366

Rahimi, F., Roshanfekr, H. and Peyman, H., 2021. Ultra-sensitive electrochemical aptasensor for label-free detection of aflatoxin $B_{1}$ in wheat flour sample using factorial design experiments. Food Chemistry 343: 128436. https://doi.org/10.1016/j. foodchem.2020.128436 
Rämö, S., Haapalainen, M. and Latvala, S., 2021. Development and validation of a UHPLC-MS/MS method for the analysis of Fusarium mycotoxins in onion. Food Analytical Methods 14: 1524-1536. https://doi.org/10.1007/s12161-021-01992-8

Rausch, A., Brockmeyer, R. and Schwerdtle, T., 2021. Development and validation of a liquid chromatography tandem mass spectrometry multi-method for the determination of 41 free and modified mycotoxins in beer. Food Chemistry 338: 127801. https://doi. org/10.1016/j.foodchem.2020.127801

Righetti, L., Dreolin, N., Celma, A., McCullagh, M., Barknowitz, G., Sancho, J.V. and Dall'Asta, C., 2020. Travelling wave ion mobilityderived collision cross section for mycotoxins: Investigating interlaboratory and interplatform reproducibility. Journal of Agricultural and Food Chemistry 68: 10937-10943. https://doi. org/10.1021/acs.jafc.0c04498

Rivas Casado, M., Parsons, D.J., Weightman, R.M., Magan, N. and Origgi, S., 2009. Geostatistical analysis of the spatial distribution of mycotoxin concentration in bulk cereals. Food Additives and Contaminants Part A 26: 867-873. http://dx.doi. org $/ 10.1080 / 02652030902788912$

Rocchetti, G., Ghilardelli, F., Masoero, F. and Gallo, A., 2021. Screening of regulated and emerging mycotoxins in bulk milk samples by high-resolution mass spectrometry. Foods 10: 2025. https://doi. org/10.3390/foods10092025

Saito, K., Ishii, J., Naniwa, M., Ishii, R., Kato, M., Kondo, T., Sakurai, H., Taniguchi, M., Hashiguchi, S., Hayashi, T. and Ito, R., 2020. Residual analysis of aflatoxins in spice by HPLC coupled with solid-phase dispersive extraction and solid-phase fluorescence derivatization method. Journal of AOAC International 103: 1521-1527. https:// doi.org/10.1093/jaoacint/qsaa077

Santovito, E., Greco, D., D’Ascanio, V., Sanzani, S. M. and Avantaggiato, G., 2020. Development of a DNA-based biosensor for the fast and sensitive detection of ochratoxin A in urine. Analytica Chimica Acta 1133: 20-29. https://doi.org/10.1016/j.aca.2020.07.078

Scheibenzuber, S., Dick, F., Asam, S. and Rychlik, M., 2021. Analysis of 13 Alternaria mycotoxins including modified forms in beer. Mycotoxin Research 37: 149-159. https://doi.org/10.1007/s12550021-00424-0

Schmidt, J., Cramer, B., Turner, P., Stoltzfus, R.J., Humphrey, J.H., Smith, L.E. and Humpf, H.-U., 2021. Determination of urinary mycotoxin biomarkers using a sensitive online solid phase extraction-UHPLC-MS/MS method. Toxins 13: 418. https://doi. org/10.3390/toxins13060418

Steiner, D., Malachová, A., Sulyok, M. and Krska, R., 2021. Challenges and future directions in LC-MS-based multiclass method development for the quantification of food contaminants. Analytical and Bioanalytical Chemistry 413: 25-34. https://doi.org/10.1007/ s00216-020-03015-7

Steiner, D., Sulyok, M., Malachová, A., Mueller, A. and Krska, R., 2020. Realizing the simultaneous liquid chromatography-tandem mass spectrometry based quantification of $>1200$ biotoxins, pesticides and veterinary drugs in complex feed. Journal of Chromatography A 1629: 461502. https://doi.org/10.1016/j.chroma.2020.461502
Sulyok, M., Stadler, D., Steiner, D. and Krska, R., 2020. Validation of an LC-MS/MS-based dilute-and-shoot approach for the quantification of $>500$ mycotoxins and other secondary metabolites in food crops: challenges and solutions. Analytical and Bioanalytical Chemistry 412: 2607-2620. https://doi.org/10.1007/s00216-020-02489-9

Tangni, E.K., Van Hove, F., Huybrechts, B., Masquelier, J., Vandermeiren, K. and Van Hoeck, E., 2021. Citrinin determination in food and food supplements by LC-MS/MS: development and use of reference materials in an international collaborative study. Toxins 13: 245 . https://doi.org/10.3390/toxins 13040245

Thompson, M., 2000. Recent trends in inter-laboratory precision at $\mathrm{ppb}$ and sub-ppb concentrations in relation to fitness for purpose criteria in proficiency testing. Analyst 125: 385-386. https://doi. org/10.1039/b000282h

Tittlemier, S. A., Brunkhorst, J., Cramer, B., DeRosa, M. C., Lattanzio, V. M. T., Malone, R., Maragos, C., Stranska, M. and Sumarah, M. W., 2021. Developments in mycotoxin analysis: an update for 20192020. World Mycotoxin Journal 14: 3-26 https://doi.org/10.3920/ WMJ2020.2664

Tittlemier, S. A., Cramer, B., Dall'Asta, C., Iha, M.H., Lattanzio, V. M. T., Maragos, C., Solfrizzo, M., Stranska, M., Stroka, J. and Sumarah, M., 2020. Developments in mycotoxin analysis: an update for 20182019. World Mycotoxin Journal 13: 3-24 https://doi.org/10.3920/ WMJ2019.2535

Uhlig, S., Rangel-Huerta, O.D., Divon, H.H., Rolén, E., Pauchon, K., Sumarah, M.W., Vrålstad, T. and Renaud, J.B., 2021. Unraveling the ergot alkaloid and indole diterpenoid metabolome in the Claviceps purpurea species complex using LC-HRMS/MS diagnostic fragmentation filtering. Journal of Agriculture and Food Chemistry 69: 7137-7148. https://doi.org/10.1021/acs.jafc.1c01973

Vidal, A., Belova, L., Stove, C., De Boevre, M. and De Saeger, S., 2021. Volumetric absorptive microsampling as an alternative tool for biomonitoring of multi-mycotoxin exposure in resource-limited areas. Toxins 13: 345. https://doi.org/10.3390/toxins13050345

Wang, C., Zhang, L., Luo, J., Qin, J., Jiang, J., Qin, L., Zhao, Z., Yang, S. and Yang, M., 2021. Development of a sensitive indirect competitive enzyme-linked immunosorbent assay for high-throughput detection and risk assessment of aflatoxin $\mathrm{B}_{1}$ in animal-derived medicines. Toxicon 197: 99-105. https://doi.org/10.1016/j.toxicon.2021.04.009

Wang, J., Chen, Q., Jin, Y., Zhang, X., He, L., Zhang, W. and Chen, Y. 2020. Surface enhanced Raman scattering-based lateral flow immunosensor for sensitive detection of aflatoxin $M_{1}$ in urine. Analytica Chimica Acta 1128: 184-192. https://doi.org/10.1016/j. aca.2020.06.076

Wood, J.E., Gill, B.D., Indyk, H.E., Rhemrev, R., Pazdanska, M., Mackay, N. and Marley, E., 2020. Determination of aflatoxin $M_{1}$ in liquid milk, cheese, and selected milk proteins by automated online immunoaffinity cleanup with liquid chromatography-fluorescence detection. Journal of AOAC International 104: 719-724. https://doi. org/10.1093/jaoacint/qsaa164

Wu, Y., Yu, B., Cui, P., Yu, T., Shi, G. and Shen, Z. 2021. Development of a quantum dot-based lateral flow immunoassay with high reaction consistency to total aflatoxins in botanical materials. Analytical Bioanalytical Chemistry 413: 1629-1637. https://doi.org/10.1007/ s00216-020-03123-4. 
Xing, C., Dong, X., Xu, T., Yuan, J., Yan, W., Sui, X. and Zhao, X., 2020. Analysis of multiple mycotoxins-contaminated wheat by a smart analysis platform. Analytical Biochemistry 610: 113928. https:// doi.org/10.1016/j.ab.2020.113928

Xiong, Z., Wang, Q., Xie, Y., Li, N., Yun, W. and Yang, L., 2021. Simultaneous detection of aflatoxin $B_{1}$ and ochratoxin $A$ in food samples by dual DNA tweezers nanomachine. Food Chemistry 338: 128122. https://doi.org/10.1016/j.foodchem.2020.128122

Xu, J., Liu, T., Chi, J., Zhang, W., Lin, C., Lin, X. and Xie, Z., 2020. Online high-efficient specific detection of zearalenone in rice by using high-loading aptamer affinity hydrophilic monolithic column coupled with HPLC. Talanta 219: 121309. https://doi.org/10.1016/j. talanta.2020.121309

Xuan, Z., Liu, H., Ye, J., Li, L., Tian, W. and Wang, S., 2020. Reliable and disposable quantum dot-based electrochemical immunosensor for aflatoxin $B_{1}$ simplified analysis with automated magneto-controlled pretreatment system. Analytical Bioanalytical Chemistry 412: 76157625. https://doi.org/10.1007/s00216-020-02897-x

Yi, Y., Fan, K., Wang, J., Fu, Q., Zhou, X., Zhang, Y. and Zhang, H., 2021. Primary research on sampling scheme for analyzing mycotoxin distribution in wheat and rice fields. Journal of the Science of Food and Agriculture 101: 4980-4986. https://doi.org/10.1002/jsfa.11141
Zachariasova, M., Cuhra, P. and Hajslova, J., 2014. Cross-reactivity of rapid immunochemical methods for mycotoxins detection towards metabolites and masked mycotoxins: the current state of knowledge. World Mycotoxin Journal 7: 449-464. https://doi.org/10.3920/ WMJ2014.1701

Zangheri, M., Di Nardo, F., Calabria, D., Marchegiani, E., Anfossi, L., Guardigli, M., Mirasoli, M., Baggiani, C. and Roda. C., 2021. Smartphone biosensor for point-of-need chemiluminescence detection of ochratoxin A in wine and coffee. Analytica Chimica Acta 1163: 338515. https://doi.org/10.1016/j.aca.2021.338515

Zhang, L., Zhang, Z., Tian, Y., Cui, M., Huang, B., Luo, T., Zhang, S. and Wang, H., 2021b. Rapid, simultaneous detection of mycotoxins with smartphone recognition-based immune microspheres. Analytical Bioanalytical Chemistry 413: 3683-3693. https://doi.org/10.1007/ s00216-021-03316-5

Zhang, X., Qu, J., Dai, Z., Lin, Y., Lu, G., Yang, S., You, Y., Liu, H., Wu, Y. and Jiang, G., 2021a. Data-dependent acquisition based highresolution mass spectrum for trace Alternaria mycotoxin analysis and sulfated metabolites identification. Food Chemistry 364: 130450. https://doi.org/10.1016/j.foodchem.2021.130450 


\section{Appendix. List of abbreviations}

\begin{tabular}{|c|c|c|c|}
\hline 3-ADON & 3-acetyl-deoxynivalenol & LFIA & lateral flow immunoassay \\
\hline 15-ADON & 15-acetyl-deoxynivalenol & LOD & limit of detection \\
\hline ag & attogram & LOQ & limit of quantitation \\
\hline AIF & all ion fragmentation & mAbs & monoclonal antibodies \\
\hline $\mathrm{AFB}_{1}$ & aflatoxin $\mathrm{B}_{1}$ & MIPs & molecularly imprinted polymers \\
\hline $\mathrm{AFB}_{2}$ & aflatoxin $\mathrm{B}_{2}$ & MGNH & magneto-gold nanohybrid \\
\hline $\mathrm{AFG}_{1}$ & aflatoxin $\mathrm{G}_{1}$ & MS & mass spectrometry \\
\hline $\mathrm{AFG}_{2}$ & aflatoxin $\mathrm{G}_{2}$ & MON & moniliformin \\
\hline $\mathrm{AFM}_{1}$ & aflatoxin $\mathrm{M}_{1}$ & MS/MS & tandem mass spectrometry \\
\hline $\mathrm{AFM}_{2}$ & aflatoxin $\mathrm{M}_{2}$ & nanoMIPs & molecularly imprinted polymer nanoparticles \\
\hline AME & alternariol monomethyl ether & NIR & near-infrared \\
\hline $\mathrm{AOH}$ & alternariol & NIV & nivalenol \\
\hline AuNP & colloidal gold nanoparticles & $\mathrm{nm}$ & nanometres \\
\hline BEA & beauvericin & NTA & non-targeted analysis \\
\hline BSA & bovine serum albumin & $\mathrm{OT} \alpha$ & ochratoxin $\alpha$ \\
\hline CCS & collisional cross section & OTA & ochratoxin A \\
\hline CIT & citrinin & PAT & patulin \\
\hline CTV & citreoviridin & PDA & photodiode array detection \\
\hline CRM & certified reference material & PT & proficiency test \\
\hline CPA & cyclopiazonic acid & Q-ToF & quadrupole-time of flight \\
\hline DDA & data-dependent acquisition & QD & quantum dot \\
\hline DIA & data-independent acquisition & QuEChERS & quick, easy, cheap, effective, rugged, and safe \\
\hline $\mathrm{DON}$ & deoxynivalenol & $\mathrm{RCA}$ & rolling circle amplification \\
\hline DON-3G & deoxynivalenol-3-glucoside & $\mathrm{ROC}$ & roquefortine $\mathrm{C}$ \\
\hline dSPE & dispersive solid phase extraction & RSD & relative standard deviation \\
\hline ELISA & enzyme-linked immunosorbent assay & SERS & surface-enhanced Raman spectroscopy \\
\hline ELASA & enzyme-linked apta-sorbent assay & SPE & solid-phase extraction \\
\hline $\mathrm{EU}$ & European Union & SPR & surface plasmon resonance \\
\hline $\mathrm{FB}_{1}$ & fumonisin $B_{1}$ & $\mathrm{~T}-2$ & $\mathrm{~T}-2$ toxin \\
\hline $\mathrm{FB}_{2}$ & fumonisin $\mathrm{B}_{2}$ & TeA & tenuazonic acid \\
\hline $\mathrm{FB}_{3}$ & fumonisin $\mathrm{B}_{3}$ & UHPLC & ultra-high pressure liquid chromatography \\
\hline FLD & fluorescence detection & UV & ultraviolet \\
\hline FPIA & fluorescence polarization immunoassay & VAMS & volumetric absorptive microsampling \\
\hline HPLC & high-performance liquid chromatography & $\mathrm{v} / \mathrm{v}$ & volume/volume \\
\hline HRMS & high resolution mass spectrometry & $\mathrm{ZAN}$ & zearalanone \\
\hline HT-2 & HT-2 toxin & $\mathrm{ZEN}$ & zearalenone \\
\hline IAC & immunoaffinity chromatography & $\alpha-Z E L$ & $\alpha$-zearalenol \\
\hline $\mathrm{IC}_{50}$ & half maximal inhibitory concentration & $\beta-Z E L$ & $\beta$-zearalenol \\
\hline
\end{tabular}


